Research Article

Published May 8, 2020

\title{
Passive Immunity to Vibrio cholerae O1 Afforded by a Human Monoclonal IgA1 ANTIbOdy EXPRESSED In MiLK
}

\section{AUTHORS}

Danielle E. Baranova ${ }^{1,2}$, Lihow Chen $^{3}$, Margaret Destrempes ${ }^{3}$, Harry Meade ${ }^{3}$, Nicholas J. Mantis ${ }^{1,2}$

\section{AFFILIATED INSTITUTIONS}

${ }^{1}$ Department of Biomedical Sciences; University at Albany; Albany, New York

${ }^{2}$ Division of Infectious Diseases; Wadsworth Center; New York State Department of Health; Albany, New York

${ }^{3}$ LFB USA; Framingham, Massachusetts

\section{CORRESPONDING AUTHOR}

Nicholas J. Mantis

Division of Infectious Diseases

Wadsworth Center

New York State Department of Health

120 New Scotland Ave.

Albany, NY 12208

(518) 473-7487

nicholas.mantis@health.ny.gov

\section{SUGGESTED CITATION}

Baranova DE, Chen L, Destrempes M, Meade H, Mantis NJ. Passive Immunity to Vibrio cholerae O1 Afforded by a Human Monoclonal IgA1 Antibody Expressed in Milk. Pathogens and Immunity. 2020;5(1):89-116. doi: 10.20411/pai.v5i1.370

\section{ABSTRACT}

Background: In cholera epidemics, the spread of disease can easily outpace vaccine control measures. The advent of technologies enabling the expression of recombinant proteins, including antibodies, in the milk of transgenic animals raises the prospect of developing a self-administered and cost-effective monoclonal antibody (MAb)-based prophylactic to reduce the incidence of Vibrio cholerae infection. 
Methods: We generated a transgenic mouse line in which the heavy and light chain variable regions (Fv) specific for a conserved epitope in the core/lipid A of $V$. cholerae $\mathrm{O} 1$ lipopolysaccharide were expressed as a full-length human dimeric IgA1 (ZAC-3) and secreted into the milk of lactating dams. Milk containing ZAC-3 IgA1 was assessed for the ability to passively protect against experimental cholera infection in a newborn mouse model and to impact bacterial swimming behavior.

Results: Newborn mice that were passively administered ZAC-3 IgA1 containing milk, or that suckled on dams expressing ZAC-3 IgA1, were immune to experimental cholera infection, as measured by a reduction of $V$. cholerae $\mathrm{O} 1$ colony forming units recovered from intestinal lysates 12 hours after oral challenge. In vitro analysis revealed that ZAC-3 hIgA1-containing milk arrested $V$. cholerae motility in soft agar and liquid media and was effective at promoting bacterial agglutination, possibly accounting for the observed reduction in bacterial colonization in vivo.

Conclusions: These results demonstrate that consumption of milk-derived antibodies may serve as a strategy to passively protect against cholera and possibly other enteric pathogens.

Keywords: enteric; immunity; cholera; antibody; vaccine; mucosal; milk

\section{INTRODUCTION}

Cholera is a severe diarrheal disease that can cause death within hours in the absence of intravenous rehydration therapy [1]. The disease is distributed globally with the highest incidence occurring in association with regional outbreaks. In the past 3 years in Yemen alone, there have been more than 2 million cases of cholera and more than 3,800 deaths [2]. The etiological agent of this disease is the Gram-negative bacterium, Vibrio cholerae. The bacterium is transmitted through the consumption of contaminated water and food, with the spread of disease exacerbated by breakdowns in municipal infrastructures due to natural disasters or civil conflicts. Upon entering the digestive tract, $V$. cholerae utilizes a single polar flagellum to reach the proximal small intestine, where it penetrates the viscous mucus layer overlying the epithelium, and gains access to villus crypts. Interaction with the intestinal epithelium is mediated by the toxin-coregulated pilus (TCP). Following intestinal colonization, the bacterium secretes cholera toxin (CT), an ADP-ribosylating toxin that triggers severe watery diarrhea (rice water stool) that is the hallmark of the disease and the main driver of mortality []ㅡ.

$V$. cholerae species are divided into over 200 serogroups defined by their O-polysaccharide (OPS) antigen, although only the O1 and O139 serogroups are known to cause epidemics. Since 1817 there have been 7 cholera pandemics. The first 6 pandemics were attributed to the classical biotype of $V$. cholerae $\mathrm{O} 1$, while the current pandemic is caused predominantly by the El Tor biotype $[\underline{4}, \underline{5}]$. The V. cholerae O1 classical and El Tor biotypes differ in several important respects, including the ability of most El Tor isolates to outcompete classical isolates both in vitro and in vivo. El Tor strains also have an increased ability to transition between highly motile, planktonic forms and non-motile, biofilm states, leading to increased fitness in marine environments [ㅁ-ㅁ], Additionally, due to differences in the source of bacteriophage encoding CT and the regulation of key virulence genes, classical strains secrete more CT and cause more severe disease in humans $[\underline{1}, \underline{6}$, 9]. Interestingly, there are circulating variant El Tor strains which possess the CT genetic element from classical strains, and they cause more severe disease than their predecessor seventh pandemic El Tor strains, which is thought to be due to this difference in CT genetic element source [10]. 
Individuals who experience an episode of cholera develop serotype-specific IgG and IgA antibodies in serum and intestinal secretions, respectively. The bulk of the human antibody response is directed against 2 targets: CT and the OPS of lipopolysaccharide (LPS) [11]. In terms of immunity, protection is associated with anti-OPS antibodies, not anti-CT antibodies [12-17]. Anti-CT serum and mucosal antibodies are apparently ineffective at protecting against disease because the toxin is released from the bacterium directly onto the epithelium, with little opportunity for antibodies to interfere with toxin binding or uptake [14]. Anti-LPS IgA antibodies, on the other hand, interfere with the earliest steps in V. cholerae infection. Specifically, antibodies against OPS or the core/lipid A region of LPS (see below) have been shown to arrest $V$. cholerae motility and promote bacterial agglutination [ $\underline{18}-\underline{25}]$. a severe diarrheal disease that remains endemic in many parts of the world and can cause outbreaks wherever sanitation and clean water systems break down. Prevention of disease could be achieved through improved sanitation and clean water provision supported by vaccination. $V$. cholerae serogroup $\mathrm{O} 1$ is the major cause of cholera; $\mathrm{O} 1$ serotypes Inaba and Ogawa have similar disease burdens, while $\mathrm{O} 139$ is the only non-O1 serogroup to cause epidemics. We showed previously that immunization of adult female mice with purified $V$. cholerae outer membrane vesicles (OMVs. In liquid culture, for example, V. cholerae stops swimming within minutes of being treated with LPS-specific polyclonal or monoclonal antibodies (MAb). Agglutination is the result of the formation of large macroscopic aggregates that are likely entrapped within intestinal mucus and cleared from the gut through a process known as immune exclusion [르].

Despite the success of oral cholera vaccines (OCV) in regions where cholera is endemic, there remains a need for an effective pre-exposure prophylactic (PrEP) that could be administered to individuals in outbreak situations where vaccine implementation is not immediately possible. One possible avenue is oral passive immunization with polyclonal or monoclonal antibodies. In the case of enterotoxigenic Escherichia coli (ETEC), it has been demonstrated in Phase 1 clinical trials that repeated oral delivery of hyperimmune bovine colostrum (HBS) affords protection against experimental traveler's diarrhea [27]. In the case of cholera, there is considerable evidence that anti-LPS IgA antibodies are protective when passively administered to newborn mice in an experimental cholera challenge model $[\underline{14}, \underline{17}, \underline{18}]$. In humans, there is also literature indicating that anti-LPS IgA antibodies in breast milk protect against disease [28]. Thus, platforms such as directed expression of MAb in milk of transgenic animals might be ideal for cholera applications [29].

As a proof of principle, we sought to test whether a recombinant anti-LPS IgA MAb expressed in milk can afford protection against experimental cholera challenge in neonatal mice. The MAb of choice for these studies was ZAC-3 IgA. ZAC-3 targets an epitope within the core/lipid A region of LPS that is conserved among $V$. cholerae $\mathrm{O} 1$ classical and El Tor isolates [22, $\underline{30}, \underline{31}$. ZAC3 , as either an IgA or IgG, is a particularly potent inhibitor of $V$. cholerae motility in liquid and semi-solid media. ZAC-3 also promotes $V$. cholerae agglutination and stimulates the bacteria to secrete an extracellular matrix (ECM) reminiscent of the early stages of biofilm formation [32].

In this study, we generated transgenic mice in which ZAC-3 was secreted into mouse mammary glands and secreted into the milk of lactating dams as a full-length human dimeric IgA1. In the newborn mouse model of cholera we show that milk containing ZAC-3 hIgA1 significantly reduced $V$. cholerae $\mathrm{O} 1$ colonization of the intestinal mucosa. In vitro, milk containing ZAC-3 
hIgA1 curtailed $V$. cholerae motility in soft agar and liquid media and was effective at promoting bacterial agglutination, possibly accounting for the observed reduction in bacterial colonization in vivo. These results demonstrate that consumption of milk-derived antibodies may serve as a strategy to passively protect against cholera and possibly other enteric pathogens.

\section{MATERIALS AND METHODS}

\section{Growth conditions for bacterial strains}

The bacterial strains used in this study are described in Supplemental Table 1. Bacteria were grown in $\mathrm{LB}$ medium at $37^{\circ} \mathrm{C}$ with aeration, $200 \mathrm{rpm}$. As necessary, media were supplemented with $100 \mu \mathrm{g} / \mathrm{mL}$ of streptomycin or $10 \mu \mathrm{g} / \mathrm{mL}$ of gentamicin.

\section{Generation of fluorescent $\boldsymbol{V}$. cholerae}

A high copy plasmid was engineered to constitutively express mCherry in $V$. cholerae. To accomplish this, the mCherry open reading frame was first amplified from pMQCherry80 (Matthew Wargo, unpublished) using Q5 DNA polymerase (NEB) and the xfp_ORF_SOE_F2 and mCherry_R_HindIII primer set. A constitutive derivative of the Plac promoter (PA/01/04/03) was similarly amplified from pUC18-mTn7T-eyfp-Gm [33] with the PA1/04/03_ORF_SOE_R1 and PA1/04/03_F_KpnI primer set. The resulting mCherry and PA/01/04/03 fragments were gel extracted using Thermo Scientific GeneJet kit (Cat. No. K0502) and fused together through overlap extension PCR using Q5 DNA polymerase (NEB, Cat. No. M0492S) and the PA1/04/03_F_KpnI forward and mCherry_R_HindIII reverse primers to create PA/01/04/03::mcherry. The fusion product was subsequently digested with KpnI and HindIII (NEB), ligated into similarly cut pUC18T-mTn7T-eyfp (replacing the eyfp ORF and associated promoter) [33], transformed into NEB $5 a$ chemically competent cells, and plated on LB agar supplemented with $10 \mu \mathrm{g} / \mathrm{mL}$ of gentamicin. Plasmid DNA was harvested from the gentamicin-resistant colonies that emerged, using miniprep (Qiagen), and then screened by restriction digest for the presence of the reporter gene. The resulting plasmid, pGW104, was then transformed into V. cholerae O395 using electroporation to create DB364. Primer sequences are listed in Supplemental Table 2.

\section{Monoclonal antibodies and cell lines}

Monoclonal recombinant human ZAC-3 IgG1 was used as a reference [34]. Human IgA from colostrum was used a positive control in IgA sandwich ELISAs (RRID:AB_1163623). Goat anti-human IgG-HRP (RRID:AB_228265) and a goat anti-human IgA HRP conjugate (MP Biomedicals) secondary antibodies were utilized in ELISAs.

\section{Construction and production of ZAC-3 IgA1 HC, LC, and J chain}

The beta casein expression vectors contain $6.1 \mathrm{~kb}$ of upstream promoter sequence linked to an XhoI cloning site, located just before the translation start of the beta casein coding sequence. Downstream is either a $7.2 \mathrm{~kb}$ downstream sequence including the last 3 exons of beta casein (BC350, BC451) or the $300 \mathrm{bp}$ bgH polyA sequence, (BC2797). There is a $2.4 \mathrm{~kb}$ sequence encoding the chicken beta globin hypersensitive site that is positioned upstream of the casein promoter. The amino acid sequence of the ZAC-3 variable regions was taken from a previous publication [34]. The heavy chain (HC) variable region was successfully used to generate $\mathrm{HC}$ constructs of IgA1. The variable light chain (LC) sequence was copied and in so doing 2 amino acid sequences were omitted. The sequence carries a GS deletion at this LC sequence that was used in the 
milk expression of the IgA1 version of the ZAC3 antibody. Constructs were completed which contained the LC (BC3236), J chain (BC2635), both the LC and J chain (BC3239), and the HC (BC3242) of IgA1. These constructs are listed in Supplemental Table 3 and described in detail in Supplemental Figures 1-4. We noted after the fact that the LC sequence in BC3239 is missing 2 codons (and therefore amino acids) from the original ZAC-3 LC, although functional studies indicate that the deletion did not significantly impact ZAC-3 functionality.

\section{Construction and production of ZAC-3 IgA1 milk-specific vectors and expression in mice}

Transgenic mice were generated using BC3239 and BC3242 plasmids. The electroeluted fragments containing the eukaryotic sequences were mixed and micro-injected at $1 \mathrm{ng} / \mathrm{uL}$ using standard procedures (Supplementary Figure 5). The progeny produced were analyzed for the presence of the transgenes by Transnetyx (https://www.transnetyx.com Transnetyx Inc. Cordova, TN). The samples were analyzed by qPCR using primers specific for J chain, LC, and HC (Supplementary Table 3). Twelve mice were found to be transgenic for all 3 genes. The 6 founder females were grown to maturity, bred, and brought into lactation. Their milk was analyzed for the presence of the MAbs by western blot. One line, No.21 produced relatively higher levels of the antibody and was chosen for strain expansion (Supplementary Figure 6). From this founder mouse are the F1, No.172 and 3 F2 females No.191, 192, 196. The m137 mouse did not produce antibody, so it was used as a negative control in the following experiments.

\section{Western blotting}

Western blots were performed using goat anti-human IgA (AB_218398), with secondary rabbit anti-goat conjugated to Alexa Fluor 546 (AB_2535742), and anti-human J chain (AB_2121629) with LI-COR Biosciences anti-rabbit conjugated to IRDye 680RD secondary antibody (AB_10956166). Results confirm the presence of the HC (Supplementary Figure 6A), LC (Supplementary Figure 6B), and J chain (Supplementary Figure 6C).

\section{Bacterial agglutination assays}

Agglutination assays were performed as described at the following link dx.doi.org/10.17504/protocols.io.baahiab6 [23].

\section{ELISAs}

Variations on the ELISA plate set up and antibodies used are described below. All ELISAs utilized Immunolon ${ }^{\mathrm{TM}}$ 4HBX 96-well microtiter ELISA plates and were developed using SureBlue ${ }^{\mathrm{TM}}$ Microwell Peroxidase Substrate. Plates were analyzed using a Spectromax 250 spectrophotometer with Softmax Pro 5.0 software (Molecular Devices).

Whole bacteria ELISAs were performed as previously described at the following link $\underline{\mathrm{dx}}$.doi. org/10.17504/protocols.io.baajiacn [프] .

An IgA sandwich ELISA was utilized to determine IgA concentration in milk. Plates were coated overnight with $1 \mu \mathrm{g} / \mathrm{mL}$ of unlabeled Goat anti-human IgA (a-chain specific). Milk samples were serially diluted across plates, with human IgA from colostrum (Sigma-Aldrich Cat. No. I2636, RRID:AB_1163623) used as a control. A goat anti-human serum IgA conjugated to HRP secondary antibody was added at a 1:5,000 dilution to all wells (MP Biomedicals). For experiments testing the IgA concentration in the stomach contents of pups, we utilized this same assay, with 
the stomach contents mixed with protease inhibitor cocktail, described below, as the primary antibody.

For ZAC-3 IgG competition ELISAs, plates were coated with whole V. cholerae O395 cells as described above. All wells were subsequently coated with $0.1 \mu \mathrm{g} / \mathrm{mL}$ of ZAC-3 IgG for 1 hour at room temperature. Plates were washed and IgA1-milk samples were incubated for 1 hour. A goat anti-human IgG conjugated to HRP secondary antibody was used at a 1:5,000 dilution for 1 hour (RRID:AB_2535582).

\section{Bacterial motility assays}

Liquid motility and semi-solid agar (ssAgar) assays were performed as described previously [23] .

\section{Animal care and ethics statement}

The mouse experiments described in this study were reviewed and approved by the Wadsworth Center's Institutional Animal Care and Use Committee (IACUC) under protocol \#17-428. The Wadsworth Center complies with the Public Health Service Policy on Humane Care and Use of Laboratory Animals and was issued assurance number A3183-01. The Wadsworth Center is fully accredited by the Association for Assessment and Accreditation of Laboratory Animal Care (AAALAC). Obtaining this voluntary accreditation status reflects that Wadsworth Center's Animal Care and Use Program meets all standards required by law and goes beyond these standards as it strives to achieve excellence in animal care and use. Mice were euthanized by carbon dioxide asphyxiation followed by cervical dislocation, as recommended by the Office of Laboratory Animal Welfare (OLAW), National Institutes of Health.

\section{Neonatal mouse model of cholera.}

Neonatal mouse colonization studies were performed as described [22]. Two iterations of this experiment were done. For passive protection studies, 4- to 5-day-old BALB/c mice were removed from their dams and gavaged with $50 \mu \mathrm{L}$ of $\sim 1 \times 10^{7}$ mid-log phase $V$. cholerae cells combined with the indicated milk treatment and blue food dye, utilizing a 24-gauge feeding needle (Harvard Apparatus; Cat. No. 75-0280). For experiments utilizing pups fed by IgA1 producing dams, pups were fed from control or ZAC-3 hIgAl producing dams and gavaged with $V$. cholerae mixed with blue food dye only. At the time of gavage, 2 pups from every litter were euthanized, stomach and intestines taken, and homogenized as described below in $250 \mu \mathrm{L}$ of PBS containing cOmplete, Mini, EDTA-free Protease Inhibitor Cocktail Tablets (Sigma Aldrich, Cat. No. 11836170001). Stomach and Intestinal contents from these pups were utilized in subsequent ELISAs and motility assays, as described previously. For all experiments gavaged pups were kept at $30^{\circ} \mathrm{C}$ for 24 hours, euthanized via decapitation with sharp scissors, and whole intestines homogenized in $2 \mathrm{~mL}$ non-reinforced tubes (Fisher Scientific, Cat. No. 15-340-161) containing 4 to 5 UV-sterilized zirconium ceramic oxide beads (Fisher Scientific, Cat. No. 15-340-160) and 1mL of PBS. Intestines were homogenized utilizing a Fisherbrand ${ }^{\mathrm{Tx}}$ Bead Mill 4 Homogenizer (Fisher Scientific, Cat. No. 15-340-164), run at $3 \mathrm{~m} / \mathrm{s}$ for 30 seconds, 3 times. Homogenized intestines were plated to assess CFUs on indicated media. 


\section{RESULTS}

\section{Construction of a transgenic mouse expressing functional ZAC-3 hlgA1}

The HC and LC variable sequences of ZAC-3 were cloned onto human IgA1 and $\kappa$ chain expression vectors, respectively. The BC3239 construct contains the LC and J chain sequence and the BC3242 construct contains the HC sequence (Supplementary Figures 1-4). The ZAC-3 HC and LC and $J$ chain sequences are under the control of a $\beta$-casein expression cassette, which includes the promoter and downstream untranslated region of the goat $\beta$-casein gene. The $\beta$-casein promoter is activated specifically in mammary epithelial cells during lactation, due to tissue-specific transcription factors and lactation-related hormonal requirements $[\underline{35}, \underline{36}]$. The casein hIgA1 sequences were separated from prokaryotic sequencing following cleavage via restriction endonuclease digestion from the BC3239 and BC3242 constructs. They were introduced into mouse embryos via microinjection (Supplementary Figure 5) [37]. Candidate transgenic mice were tested by PCR for the presence of both the LC-J chain cassette (BC3239) and the HC cassette (BC3242) using primers listed in Supplementary Table 3.

Milk was collected from 2 PCR-confirmed transgenic mice (m137 and m172) and tested for the presence of human IgA by western blot and in a human IgA-specific sandwich ELISA (see Materials and Methods). The mouse $\mathrm{m} 172$ was an F1 progeny of the expressing animal $\mathrm{m} 21$, shown in the western blot (Supplementary Figure 6). An overview of mating and experimental workflow is shown in Figure 1. Milk from m172 had 300 to $600 \mu \mathrm{g} / \mathrm{mL}$ of human IgA (Figure 2A), while m137 showed no detectable reactivity. Milk from m172 bound $V$. cholerae $\mathrm{O} 1$ classical Ogawa strain O395 by whole cell ELISA, demonstrating the presence of functional ZAC-3 IgA in milk (Figure 2B). We utilized a competition ELISA to further assess if ZAC-3 IgA1 retained the same epitope specificity as the parental ZAC-3 IgG construct. ZAC-3 hIgA1 milk samples significantly inhibited chimeric ZAC-3 IgG from binding to whole V. cholerae O395 cells (Figure 2D), indicating that transgenic CD-1 mice secrete human ZAC-3 hIgA1 that retains the core/lipid A epitope specificity. Finally, milk from m172 (but not 137) promoted V. cholerae O395 agglutination in vitro even at $>1: 1600$ dilution (Figure 2C). Of note, ZAC-3 hIgA1 antibody was secreted by mammary epithelial cells, not secreted across the epithelial layer. As such the secreted product is dimeric $(\mathrm{d} \operatorname{Ig} \mathrm{A})$ but not necessarily complexed with secretory component to form secretory IgA (SIgA). 


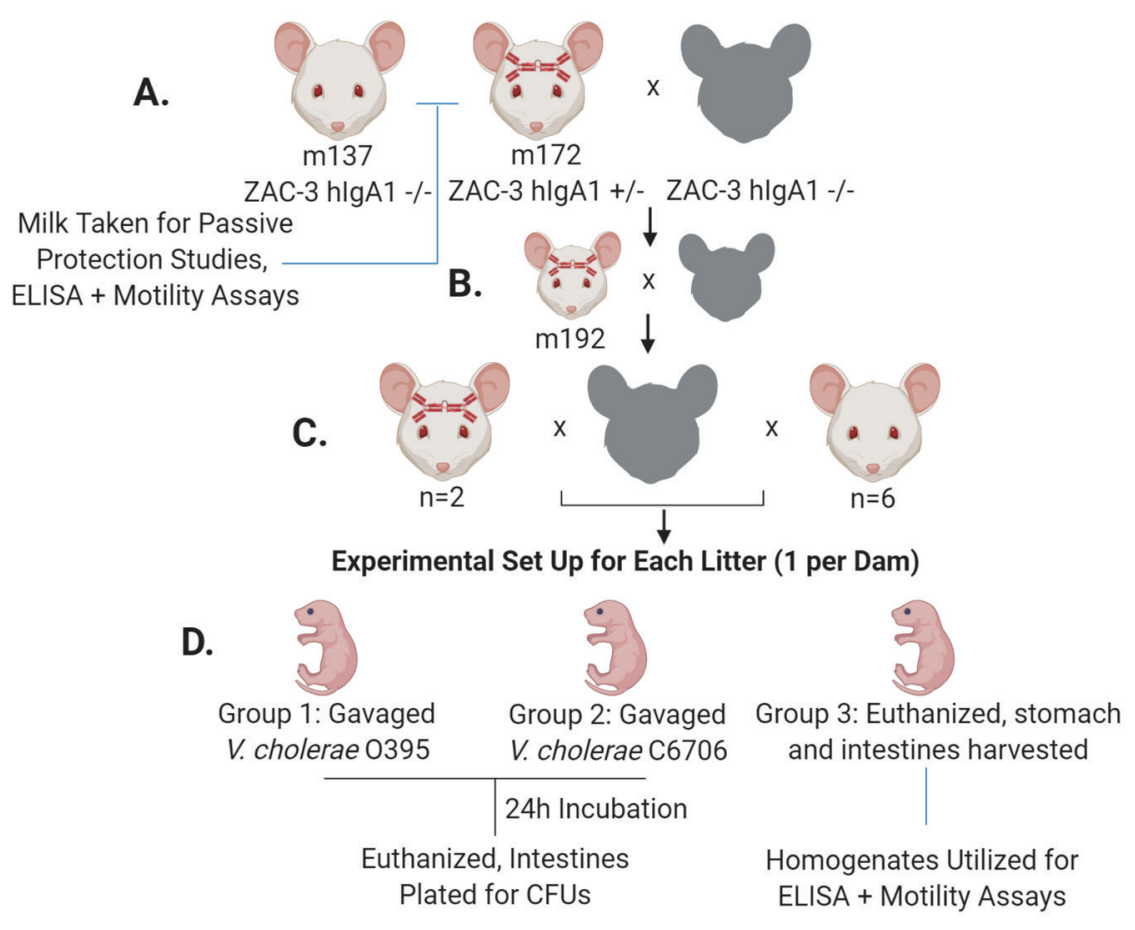

Figure 1. ZAC-3 hIgA Transgenic Mice Mating Scheme and Experimental Design. (A) Transgenic mouse m172, and a control mouse, m137, were bred with WT CD-1 males. Milk samples were taken for passive protection, ELISA and motility assays. (B) Resulting F1 progeny, m192, was bred with a WT CD-1 male. (C) From this pairing 8 F2 progeny females, 2 ZAC-3 hIgA positive, and 6 hIgA negative, were bred with WT CD-1 males. (D) F3 litters were taken at 4 to 5 days old for colonization experiments. Each litter, 1 per mating pair, was divided into 3 groups. At the time of gavage, Group 3 (1-2 pups depending on litter size) was euthanized, and stomach and intestines were excised and homogenized in PBS containing protease inhibitor. These homogenates were later used for ELISA and motility assays. Remaining litter mates were divided into Group 1 and 2, containing approximately equal numbers, and gavaged with $\sim 1 \times 10^{7} \mathrm{~V}$. cholerae O395 or C6706 strains respectively. After 24-hour incubation, pups were euthanized and whole intestines were plated onto selective media for CFUs, which serve as a surrogate for intestinal colonization of $V$. cholerae. This figure was created with www.biorender.com. 
A.

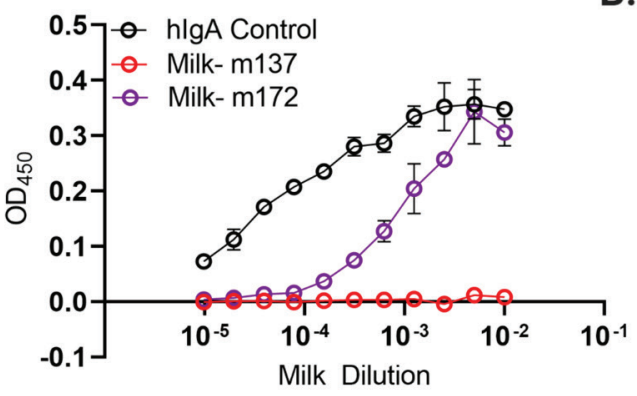

C.

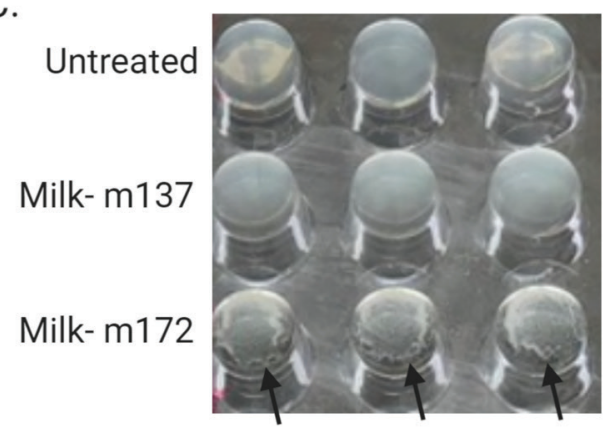

B.

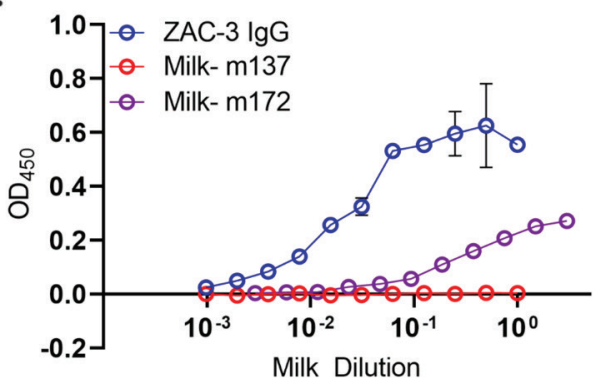

D.

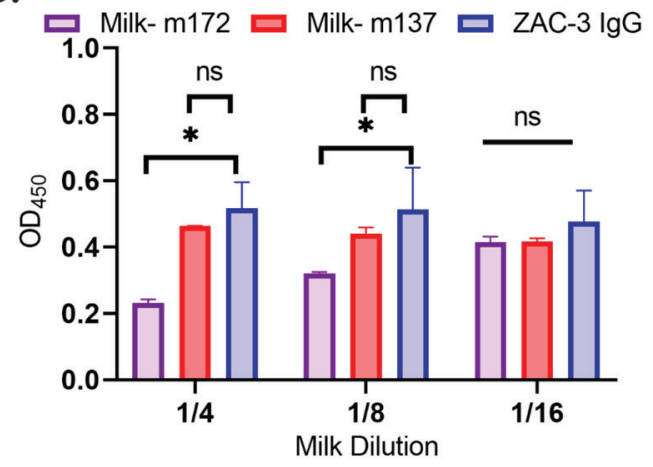

Figure 2. Transgenic Mice Secrete ZAC-3 hIgA that is Capable of Binding to and Agglutinating $V$. cholerae O1. (A) IgA sandwich ELISA to determine the concentration of hIgA in milk samples. In comparison to control human IgA from colostrum, milk from m172 exhibited above background binding to anti-human IgA ELISA plates, while milk from m132 did not. (B) Whole cell ELISA coated with $V$. cholerae $\mathrm{O} 395$ cells. Milk from m172 exhibited above background binding to V. cholerae $\mathrm{O} 395$ while milk from $\mathrm{m} 137$ did not. ZAC-3 IgG (Diluted 1:2 starting at a concentration of $10 \mu \mathrm{g} / \mathrm{mL}$ ) was included as a control for V. cholerae binding. (C) Mid-log phase V. cholerae O395 were mixed with a 1:1600 dilution of milk from either $\mathrm{m} 137$ or $\mathrm{m} 172$ for 2 hours at $37^{\circ} \mathrm{C}$ and monitored for macroagglutination. Black arrows indicate where cells treated with milk from $\mathrm{m} 172$ aggregated at the bottom of the wells, suggesting antibody in milk can crosslink V. cholerae cells and cause them to settle out of solution. (D) Competition ELISA between ZAC-3 IgG and milk containing ZAC-3 hIgA from m172 or control milk. Plates coated with whole V. cholerae $\mathrm{O} 395$ cells were incubated for 1 hour with ZAC-3 IgG at $0.1 \mu \mathrm{g} / \mathrm{mL}$, then milk from $\mathrm{m} 172$ or $\mathrm{m} 137$ was added at indicated dilutions for 1 hour. ZAC-3 IgG was detected and milk from $\mathrm{m} 172$, not $\mathrm{m} 137$, significantly inhibited binding of ZAC-3 IgG to whole V. cholerae cells, indicating that ZAC-3 hIgA can compete with ZAC-3 IgG for binding. Statistical significance was determined using 2-way ANOVA followed by Tukey multiple comparison test, ${ }^{*} P<0.05$. All data comprised at least 2 technical replicates that are representative of at least 2 biological replicates.

\section{Passive immunity afforded by milk-derived ZAC-3 hlgA1}

To test if milk-expressed ZAC-3 hIgAl affects bacterial colonization of the intestinal epithelium, we utilized the neonatal mouse model of cholera colonization [ [38]. CD-1 pups (4-5 days old) nursed by control dams or dams producing ZAC-3 hIgA1 were gavaged with either $V$. cholerae classical Ogawa O395 or El Tor Inaba strain C6706 cells. Stomach and intestinal contents from 
pups were taken at the time of gavage and tested for the presence of human IgA via ELISA to quantitate human IgA levels at the time of challenge. As determined by IgA sandwich ELISA, the stomachs and intestines isolated from pups in the ZAC-3 hIgA-positive groups contained approximately 20 to $40 \mu \mathrm{g} / \mathrm{ml}$ human IgA, while hIgA-negative groups had no detectable human IgA (Supplementary Figure 7). The stomach and intestinal homogenates from the ZAC-3 hIgA-positive groups bound whole $V$. cholerae O1 classical Ogawa strain O395 and El Tor Inaba strain C6706 by whole cell ELISA, demonstrating that ZAC-3 IgA taken in by nursing pups retains functionality and reactivity with both serotypes within the V. cholerae O1 serogroup (Supplementary Figure 8).

Eight litters of pups were gavaged with $V$. cholerae strains O395 or C6706. Among these litters, 2 were ZAC-3 hIgA positive and 6 were hIgA negative. Pups gavaged with V. cholerae strains O395 or C6706 were euthanized after 24 hours, at which time intestines were excised, homogenized, and plated on LB agar to enumerate V. cholerae CFUs as a surrogate for colonization. Pups gavaged with $V$. cholerae strains $\mathrm{O} 395$ or C6706 in the ZAC-3 hIgA-positive group showed a significant reduction in colonization in both strains in comparison to the hIgA-negative groups (Figure 3).

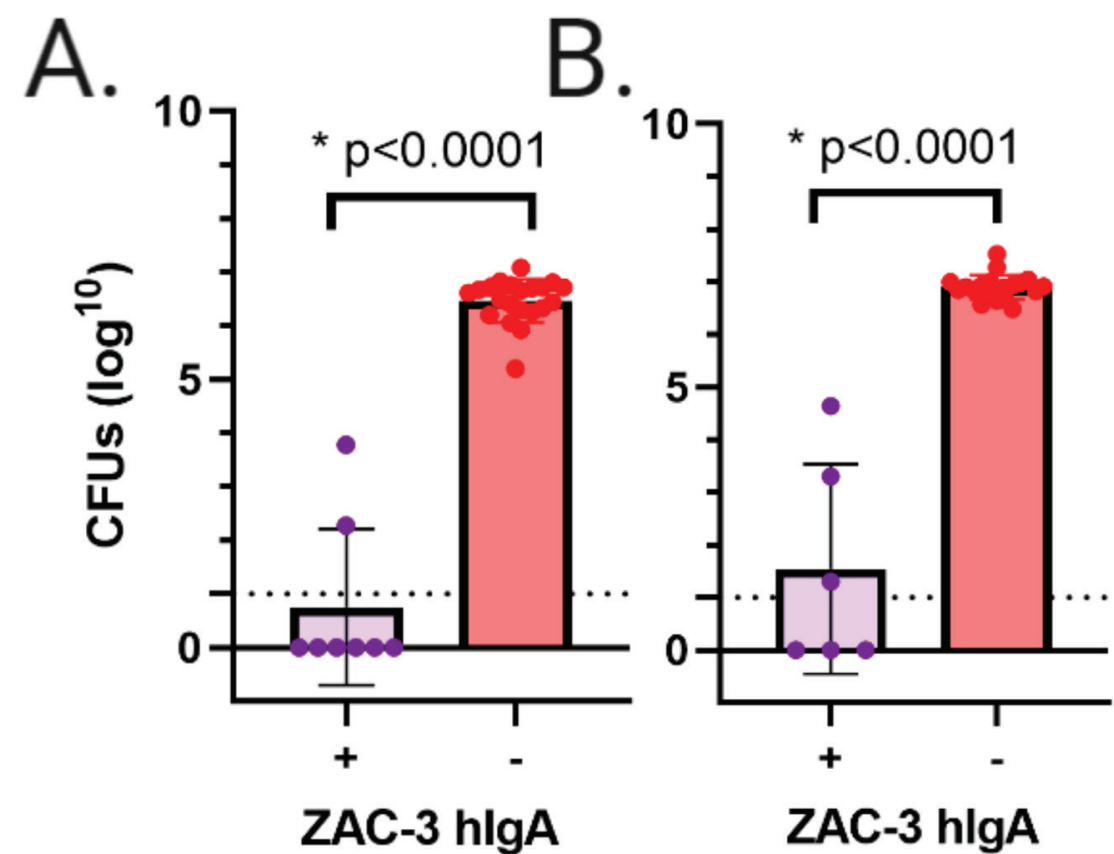

Figure 3. Milk-derived ZAC-3 hIgA1 Reduces Intestinal Colonization of V. cholerae O1. CD-1 pups, 4 to 5 days old, nursed from WT or ZAC-3 hIgA-positive transgenic dams were gavaged with $1 \times 10^{7} \mathrm{~V}$. cholerae (A) $\mathrm{O} 395$ or (B) C6706 cells. After 24 hours incubation pups were euthanized and whole intestines were plated for CFUs. There is a significant reduction in log CFUs in pups that were fed from dams secreting ZAC-3 hIgA in comparison to WT dams, for both strains of V. cholerae O1. Experiments were performed on 2 litters for hIgA positive dams, and 6 times on WT dams, with 2 to 4 pups per experimental group, each dot represents the intestinal CFUs of a single pup. Statistical significance was determined using Student's $t$-test. $P$ values are indicated on graph. 
We next performed passive transfer studies to ensure that the milk from the ZAC-3 hIgA1 transgenic dams, and not another variable, was responsible for protection against $V$. cholerae colonization. ZAC-3 hIgA1-containing milk, or control milk was diluted 1:100 (3-6 $\mu \mathrm{g} / \mathrm{mL})$, mixed with $V$. cholerae $\mathrm{O} 395$ and administered to 4 -day-old BALB/c pups. Pups treated with ZAC-3 hIgA1-containing milk exhibited a 2-log reduction of CFUs in intestinal homogenates compared to pups treated with control milk (Figure 4). Together these data show that pups fed from transgenic ZAC-3 hIgA1 dams contain milk that is positive for hIgA and exhibit decreased colonization in comparison to pups fed from hIgA-negative dams.

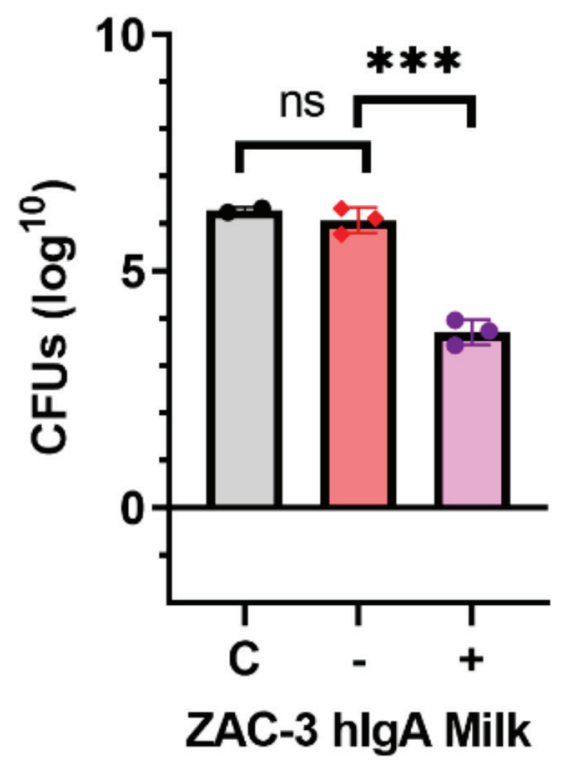

Figure 4. Pups Passively Transferred Diluted ZAC-3 hIgA-Containing Milk Exhibit Significant Reduction in $\mathbf{V}$. cholerae $\mathbf{O} 395$ Colonization. BALB/c pups, 5 days old, were orally gavaged with $1 \times 10^{7} \mathrm{~V}$. cholerae $\mathrm{O} 395$ cells premixed with a 1:100 dilution of milk from a control mouse, m137, or milk from a mouse secreting ZAC-3 hIgA1, m172. There was no significant difference in log CFUs between pups fed only $V$. cholerae alone or pups fed $V$. cholerae mixed with control milk from $\mathrm{m} 137$. There was a significant reduction in CFUs between pups fed $V$. cholerae mixed with control milk and those fed with milk from $\mathrm{m} 172$. Suggesting milk from $\mathrm{m} 172$ alone, which contains ZAC-3 hIgA1, can decrease colonization of $V$. cholerae O395. This experiment was done once, with 2 to 3 pups per experimental group as indicated on graph. Statistical significance was determined using 1-way ANOVA followed by Tukey's multiple comparison test, ${ }^{\star * *} P<0.0001$.

\section{Effect of ZAC-3 hlgA1 on V. cholerae motility}

The effect of anti-LPS IgA antibodies on $V$. cholerae colonization in the mouse model has been attributed to inhibition of flagellar motility $[\underline{18}, \underline{22}, \underline{24}]$. We therefore tested the impact of milk containing ZAC-3 hIgA1 on $V$. cholerae motility in a liquid motility assay. $V$. cholerae $\mathrm{O} 395$ cells were treated with milk from mouse 137 (negative) or mouse 172 (ZAC-3 hIgA1 positive), in liquid LB for 5 minutes, and 10 s videos were captured at the 0 and 5 -minute post-treatment time points. $V$. cholerae $\mathrm{O} 395$ cells treated with milk containing ZAC-3 hIgA1 exhibited significant decrease in motility over the course of 5 minutes (Figure 5A; Supplementary Videos 1 and 2). 
To support these results, we utilized the liquid motility assay on stomach contents from the hIgA-containing milk fed to pups, as described previously. The stomach contents from pups fed from either ZAC-3 hIgA-positive or negative dams were diluted 1:100 into LB containing midlog phase V. cholerae O395 expressing mCherry on a plasmid. We saw a reduction in motility in bacteria that were treated with stomach contents from the ZAC-3 hIgA-positive pups, in comparison to the control samples (Figure 5B, Supplementary Video 3 and 4). We then utilized the ssAgar assay, whereby stomach contents from ZAC-3 hIgA1 or controls were diluted 1:150 into 0.3\% LB agar, and colonies of $V$. cholerae $\mathrm{O} 395$ and $\mathrm{C} 6706$ were stabbed into the agar. Diameter of growth was measured over the course of 6 hours to measure the inhibition of motility. We showed that bacteria treated with stomach contents from the ZAC-3 hIgA-positive group exhibited decreased motility in comparison to milk from the control groups over the course of the 6-hour assay (Figure 5C-F). Collectively this demonstrates that milk from ZAC-3 hIgA1 transgenic animals can significantly inhibit bacterial motility.

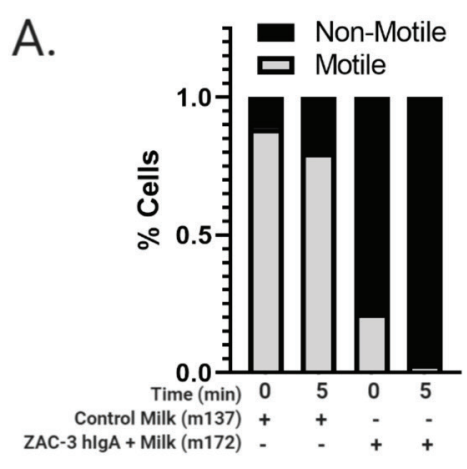

C.

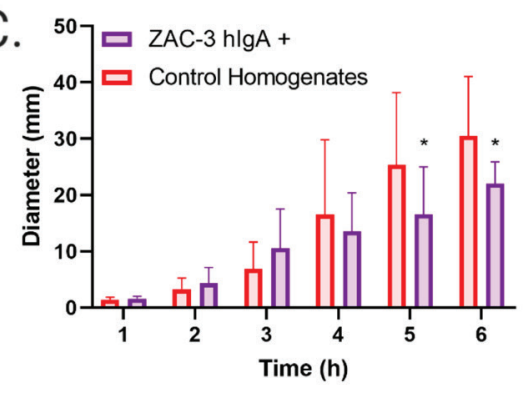

E.



B.
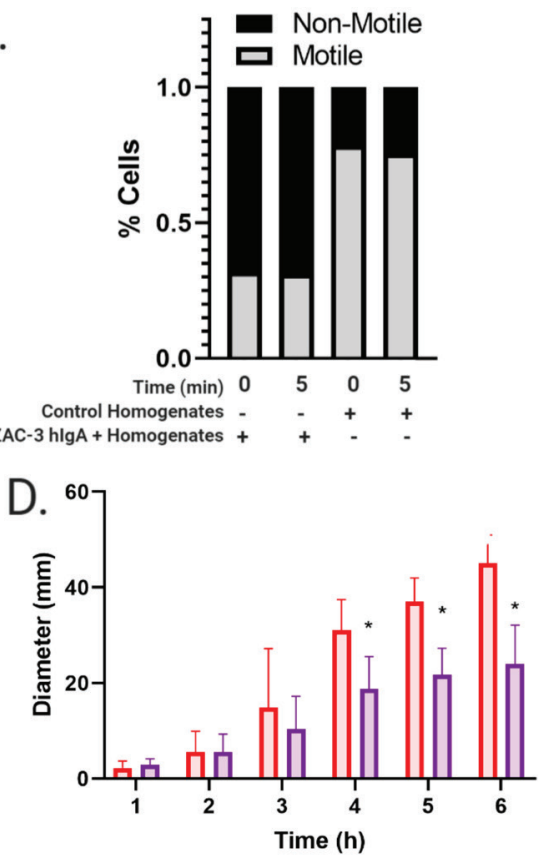

F.

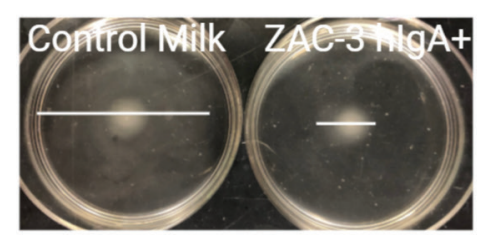

Figure 5. ZAC-3 hIgA in Milk Inhibits Motility of $\boldsymbol{V}$. cholerae O1. (A) Liquid motility assay, whereby mid-log phase $V$. cholerae $\mathrm{O} 395$ were treated with a 1:100 dilution of control milk, from m137, or ZAC-3 hIgA-containing milk from $\mathrm{m} 172$ and imaged for 10 seconds at both 0 and 5 minutes post treatment. Motile bacteria were counted as those that were seen to move between frames. There is a significant decrease $(P<0.005)$ in motility in $V$. cholerae treated with ZAC-3 hIgA-positive milk in comparison to control milk at both 0 - and 5-minutes post treatment. Data comprised 3 technical replicates. (B) Liquid motility assay was performed as described, with V. cholerae $\mathrm{O} 395$ expressing mCherry treated with either a 1:100 dilution 
of stomach homogenates from pups fed from control dams or those secreting ZAC-3 hIgA for 5 minutes. We see a significant reduction $(P<0.05)$ in motility between $V$. cholerae treated with ZAC-3 hIgA positive stomach homogenates vs control homogenates at both 0 - and 5-minutes post treatment. Data comprised at least 3 videos, from bacteria treated with at least 3 different stomach homogenates. Significance was determined using 1-way ANOVA followed by Tukey's multiple comparison test. (C, D) Semi-solid agar assay (ssAgar), whereby a colony of either V. cholerae (C) O395 or (D) C6706 from an LB agar plate was stabbed into the center of a dish containing 3\% LB agar plus either a 1:150 dilution of control or ZAC-3 hIgA-positive stomach homogenates, and the diameter of growth was measured every hour as a method to measure inhibition of motility. Cells treated with ZAC-3 hIgA-positive milk exhibited significant reduction $P<0.05)$ in motility in comparison to control milk samples at hour 5, and at hour 6 for O395 and at hours 4,5 , and 6 for C6706. Data comprised 1 biological replicate in triplicate per stomach homogenate, with at least 3 homogenates per treatment. Significance was determined using 2-way ANOVA followed by Tukey's multiple comparison test. (E, F) Representative images of 1 technical replicate from 1 treatment group at 6 hours post treatment. White lines indicate widest diameter of growth.

Lastly, it should be noted that 1 benefit of the milk-based expression system is that the MAbs are delivered along with other natural components of milk, including lactoferrin, secretory component and so on [39]. In the case of cholera, it has been reported that milk itself is able to inhibit CT binding to ganglioside (GM-1) on intestinal epithelial cells $[\underline{40}, \underline{41}]$. Indeed, we confirmed this observation in our model (Supplementary Figure 9). This is a potential added benefit of milkbased production and delivery of antibody therapy.

\section{DISCUSSION}

The current study represents a first step towards the prospect of developing a passive MAb-based oral immunization regimen as a supplement to OCV strategies, particularly in outbreak situations where cholera incidence can outpace vaccine-induced immunity. The 2 WHO pre-qualified OCVs, for example, are administered as 2 or 3 doses at approximately 2 -week intervals with overall protective efficacy ranging from $60 \%$ to $85 \%[\underline{42}, \underline{43}]$. In outbreak situations, a self-administrable, pre-exposure prophylactic could be dispensed to at-risk individuals during the days leading up to or in between OCV dosing. Theoretically, such an intervention could be implemented at the community and household levels to avert disease in particularly high-risk individuals, especially in close contact situations [ㄴ4].

Our study confirms, in a mouse model, that passive administration of anti-LPS IgA antibodies has significant benefit in terms of reducing intestinal colonization of $V$. cholerae. Anti-LPS antibodies can be either polyclonal or monoclonal in nature. For example, Bishop and colleagues demonstrated in the neonatal mouse model that protection (ie, reduced colonization) was observed when pups were suckled on dams previously vaccinated with $V$. cholerae outer membrane vesicle preparations. Immunity was associated with anti-LPS antibody titers (predominantly IgG and secondarily IgA) in milk, which in turn correlated with the ability of immune milk to arrest bacterial motility [18]. Prior to that study, Winner and colleagues demonstrated in the so-called backpack tumor model that a single IgA MAb directed against Owaga-specific epitope was protective against a lethal cholera challenge [17]. There is also evidence to suggest a benefit of passively administered anti-LPS IgA in preventing cholera in humans. Epidemiological evidence from Bangladesh reveals that the incidence of cholera is lower in breast fed children, and that the active factor in milk is primarily anti-LPS IgA [2요. 
ZAC-3, which was originally isolated from a mouse Peyer's patch-derived B-cell hybridoma, is unusual in that it is directed against an epitope within the core/lipid A region of $V$. cholerae $\mathrm{O} 1$ LPS shared across all clinical isolates we have tested [ $\underline{30}, \underline{31}]$. In humans (and mice for that matter), most anti-V. cholerae LPS antibodies target OPS [11]. However, the atypical nature of ZAC-3 should not exclude it from consideration for development as a putative prophylactic, especially considering the fact that its epitope is conserved across $V$. cholerae $\mathrm{O} 1$ serotypes [30, 32]. ZAC-3 IgG has been shown to inhibit flagella-based motility and agglutinates Classical and El Tor clinical isolates, 2 effector functions that are thought to contribute to immunity in animal models [32]. In this study, passively transferred milk containing ZAC-3 hIgA1 diluted 1:100 premixed with $V$. cholerae resulted in a $\sim 2-\log$ reduction in CFUs recovered from neonatal mouse intestines (Figure 4). In human clinical trials it has been demonstrated that even small changes in challenge dose (+/- $1 \log$ CFU) can have a significant impact on clinical presentation (eg, asymptomatic vs symptomatic) [4드. Therefore, it is likely that use of ZAC-3 hIgA1- containing milk as a prophylactic $\mathrm{MAb}$ therapy would result in a significant difference in clinical outcome in comparable human trials. In terms of scale-up and production, it has already been shown that ZAC-3 retains its biological efficacy when expressed as a recombinant human IgG1 in a Nicotiana-based platform [ $\underline{22}$, 34] and now as a human dimeric IgA1 molecule in a mammalian mammary gland system.

One caveat of our current study is that ZAC-3 IgA was expressed as a dimeric IgA1 molecule without the addition of secretory component (SC). The SC is a $\sim 75 \mathrm{kDa}$ glycoprotein derived from the pIgR that normally covalently associates with dimeric IgA during transcytosis across mucosal epithelia, including the mammary epithelium [ㄷ]. In the current model, ZAC-3 IgA was expressed by mammary epithelial cells (under control of a $\beta$-casein expression cassette) and secreted directly from the cells, independent of pIgR-mediated transport. Nonetheless, there is a small amount of free SC in breast milk, so it is conceivable that a fraction of ZAC-3 dimeric IgA1 did in fact convert to SIgA. We would expect that ZAC-3 SIgA would have additional benefits in terms of protective immunity, as SC imparts a number of unique traits upon IgA, including improved GI stability and association with mucus. SIgA may also synergize with other factors in breast milk, including lactoferrin. Finally, there are reports that human SC specifically affects $V$. cholerae biofilm formation and therefore might affect intestinal colonization [47]. It remains to be tested whether ZAC-3 SIgA would have had benefits greater than IgA alone.

In summary, we show here the effectiveness of the milk-expression platform in producing a previously characterized monoclonal antibody directed against a cross-protective epitope on $V$. cholerae O1 LPS. This work supports the milk-production platform as a viable mechanism of producing prodigious amounts of IgA, with real-world application for utilization of this platform for production in larger animals, ie, cows and goats, in order to combat many diseases of public health importance in high-risk populations.

\section{FUNDING}

Research reported in this study was supported by the National Institute of Allergy and Infectious Diseases (NIAID) of the National Institutes of Health (NIH) under award number R21-AI109275 to NJM. The content is solely the responsibility of the authors and does not necessarily represent the official views of the NIH. The funders had no role in study design, data collection and analysis, decision to publish, or preparation of the manuscript. 


\section{CONFLICTS OF INTEREST}

LC, MD, and HM are current or former employees of LFB USA, which generates transgenic mouse strains; DB performed all in vitro and in vivo studies with V. cholerae; DB, LC, HM, and NM analyzed the data; DB, HM, and NM wrote the manuscript.

\section{ACKNOWLEDGMENTS}

We thank Rich Cole of the Wadsworth Centers Advanced Light Microscopy and Image Analysis Core Facility for assistance with microscopy. We thank Dr. Graham Willsey for technical assistance and Angelene Richards for valuable feedback.

\section{REFERENCES}

1. Kaper JB, Morris JG, Levine MM. Cholera. Clin Microbiol Rev. 1995;8: 48-86.

2. World Health Organization. The Work of WHO in the Eastern Mediterranean Region Annual Report of the Regional Director. 2011. Available: http://applications.emro.who.int/docs/ RD_Annual_Report_2012_en_14587.pdf

3. Krebs SJ, Taylor RK. Protection and Attachment of Vibrio cholerae Mediated by the Toxin-Coregulated Pilus in the Infant Mouse Model. J Bacteriol. 2011;193: 5260-5270. doi:10.1128/jb.00378-11

4. Devault AM, Golding GB, Waglechner N, Enk JM, Kuch M, Tien JH, Shi M, Fisman DN, Dhody AN, Forrest S, Bos KI, Earn DJ, Holmes EC, Poinar HN. Second-pandemic strain of Vibrio cholerae from the Philadelphia cholera outbreak of 1849. N Engl J Med. 2014;370(4):334-40. PubMed PMID. doi: 10.1056/NEJMoa1308663

5. Hu D, Liu B, Feng L, Ding P, Guo X, Wang M, Cao B, Reeves PR, Wang L. Origins of the current seventh cholera pandemic. Proc Natl Acad Sci U S A. 2016;113(48):E7730-E9. PubMed PMID: PMC5137724. doi: 10.1073/pnas. 1608732113

6. Beyhan S, Tischler AD, Camilli A, Yildiz FH. Differences in gene expression between the classical and E1 Tor biotypes of Vibrio cholerae O1. Infect Immun. 2006;74: 3633-42. doi:10.1128/iai.01750-05

7. Huq MI, Sanyal SC, Samadi AR, Monsur KA. Comparative behaviour of classical and El Tor biotypes of Vibrio cholerae 01 isolated in Bangladesh during 1982. J Diarrhoeal Dis Res. 1983;1: 5-9.

8. Pradhan S, Baidya AK, Ghosh A, Paul K, Chowdhury R. The El Tor Biotype of Vibrio cholerae Exhibits a Growth Advantage in the Stationary Phase in Mixed Cultures with the Classical Biotype. J Bacteriol. 2010;192: 955-963. doi:10.1128/JB.01180-09

9. Clemens JD, Nair GB, Ahmed T, Qadri F, Holmgren J. Cholera. Lancet. 2017;390: 15391549. doi:10.1016/s0140-6736(17)30559-7 
10. Safa A, Sultana J, Cam PD, Mwansa JC, Kong RYC. Vibrio cholerae O1 Hybrid El Tor Strains, Asia and Africa. Emerg Infect Dis. 2008;14: 987-988. doi:10.3201/eid1406.080129

11. Kauffman RC, Bhuiyan TR, Nakajima R, Mayo-Smith LM, Rashu R, Hoq MR, Chowdhury F, Khan AI, Rahman A, Bhaumik SK, Harris L, O'Neal JT, Trost JF, Alam NH, Jasinskas A, Dotsey E, Kelly M, Charles RC, Xu P, Kovac P, Calderwood SB, Ryan ET, Felgner PL, Qadri F, Wrammert J, Harris JB. Single-Cell Analysis of the Plasmablast Response to Vibrio cholerae Demonstrates Expansion of Cross-Reactive Memory B Cells. mBio. 2016;7(6). PubMed PMID: PMC5181778. doi: 10.1128/mBio.02021-16

12. Aktar A, Rahman MA, Afrin S, Faruk MO, Uddin T, Akter A, Sami MIN, Yasmin T, Chowdhury F, Khan AI, Leung DT, LaRocque RC, Charles RC, Bhuiyan TR, Mandlik A, Kelly M, Kovac P, Xu P, Calderwood SB, Harris JB, Qadri F, Ryan ET. O-Specific Polysaccharide-Specific Memory B Cell Responses in Young Children, Older Children, and Adults Infected with Vibrio cholerae O1 Ogawa in Bangladesh. Clin Vaccine Immunol. 2016;23(5):427-35. PubMed PMID: PMC4860469. doi: 10.1128/CVI.00647-15

13. Ali M, Emch M, Park JK, Yunus M, Clemens J. Natural cholera infection-derived immunity in an endemic setting. J Infect Dis. 2011;204: 912-8. doi:10.1093/infdis/jir416

14. Apter FM, Michetti P, Winner LS, Mack JA, Mekalanos JJ, Neutra MR. Analysis of the roles of antilipopolysaccharide and anti-cholera toxin immunoglobulin A ( $\operatorname{Ig} \mathrm{A})$ antibodies in protection against Vibrio cholerae and cholera toxin by use of monoclonal IgA antibodies in vivo. Infect Immun. 1993;61: 5279-5285.

15. Apter FM, Lencer WI, Finkelstein RA, Mekalanos JJ, Neutra MR. Monoclonal immunoglobulin A antibodies directed against cholera toxin prevent the toxin-induced chloride secretory response and block toxin binding to intestinal epithelial cells in vitro. Infect Immun. 1993;61: 5271-8.

16. Leung DT, Rahman MA, Mohasin M, Riyadh MA, Patel SM, Alam MM, Chowdhury F, Khan AI, Kalivoda EJ, Aktar A, Bhuiyan MS, LaRocque RC, Harris JB, Calderwood SB, Qadri F, Ryan ET. Comparison of memory B cell, antibody-secreting cell, and plasma antibody responses in young children, older children, and adults with infection caused by Vibrio cholerae O1 El Tor Ogawa in Bangladesh. Clin Vaccine Immunol. 2011;18(8):131725. PubMed PMID: PMC3147357. doi: 10.1128/CVI.05124-11

17. Winner L, Mack J, Weltzin R, Mekalanos JJ, Kraehenbuhl JP, Neutra MR. New model for analysis of mucosal immunity: intestinal secretion of specific monoclonal immunoglobulin A from hybridoma tumors protects against Vibrio cholerae infection. Infect Immun. 1991;59: 977-982.

18. Bishop AL, Schild S, Patimalla B, Klein B, Camilli A. Mucosal Immunization with Vibrio cholerae Outer Membrane Vesicles Provides Maternal Protection Mediated by Antilipopolysaccharide Antibodies That Inhibit Bacterial Motility. Infect Immun. 2010;78: 4402-4420. doi:10.1128/iai.00398-10 
19. Bougoudogo F, Vely F, Nato F, Boutonnier A, Gounon P, Mazie J-C, et al. Protective activities of serum immunoglobulin $\mathrm{G}$ on the mucosal surface to Vibrio cholerae O1. Paris; 1995.

20. Gustafsson B, Holme T. Rapid detection of Vibrio cholerae O:1 by motility inhibition and immunofluorescence with monoclonal antibodies. Eur J Clin Microbiol. 1985;4: 291-4.

21. Leitner DR, Feichter S, Schild-Prüfert K, Rechberger GN, Reidl J, Schild S. Lipopolysaccharide Modifications of a Cholera Vaccine Candidate Based on Outer Membrane Vesicles Reduce Endotoxicity and Reveal the Major Protective Antigen. Infect Immun. 2013;81: 2379-2393. doi:10.1128/iai.01382-12

22. Levinson KJ, Baranova DE, Mantis NJ. A monoclonal antibody that targets the conserved core/lipid A region of lipopolysaccharide affects motility and reduces intestinal colonization of both classical and E1 Tor Vibrio cholerae biotypes. Vaccine. 2016;34: 5833-5836. doi:10.1016/j.vaccine.2016.10.023

23. Levinson KJ, De Jesus M, Mantis NJ. Rapid effects of a protective O-polysaccharide-specific monoclonal IgA on Vibrio cholerae agglutination, motility, and surface morphology. Infect Immun. 2015;83: 1674-83. doi:10.1128/iai.02856-14

24. Wang Z, Lazinski DW, Camilli A. Immunity Provided by an Outer Membrane Vesicle Cholera Vaccine Is Due to O-Antigen-Specific Antibodies Inhibiting Bacterial Motility. Infect Immun. 2016;85. doi:10.1128/IAI.00626-16

25. Dharmasena MN, Krebs SJ, Taylor RK. Characterization of a novel protective monoclonal antibody that recognizes an epitope common to Vibrio cholerae Ogawa and Inaba serotypes. Microbiology. 2009;155: 2353-2364. doi:doi:10.1099/mic.0.025726-0

26. Mantis NJ, Rol N, Corthésy B. Secretory IgA's complex roles in immunity and mucosal homeostasis in the gut. Mucosal Immunol. 2011;4: 603-611. doi:10.1038/mi.2011.41

27. Otto W, Najnigier B, Stelmasiak T, Robins-Browne RM. Randomized control trials using a tablet formulation of hyperimmune bovine colostrum to prevent diarrhea caused by enterotoxigenic Escherichia coli in volunteers. Scand J Gastroenterol. 2011;46: 862-868. doi:10.3 $109 / 00365521.2011 .574726$

28. Glass RI, Svennerholm AM, Stoll BJ, Khan MR, Hossain KM, Huq MI, Holmgren J. Protection against cholera in breast-fed children by antibodies in breast milk. N Engl J Med. 1983;308(23):1389-92. PubMed PMID. doi: 10.1056/NEJM198306093082304

29. Gavin W, Blash S, Buzzell N, Pollock D, Chen L, Hawkins N, Howe J, Miner K, Pollock J, Porter C, Schofield M, Echelard Y, Meade H. Generation of transgenic goats by pronuclear microinjection: a retrospective analysis of a commercial operation (1995-2012). Transgenic Res. 2018;27(1):115-22. PubMed PMID. doi: 10.1007/s11248-017-0050-1 
30. Wang J, Villeneuve S, Zhang J, Lei P, Miller CE, Lafaye P, Nato F, Szu SC, Karpas A, Bystricky S, Robbins JB, Kovac P, Fournier JM, Glaudemans CP. On the antigenic determinants of the lipopolysaccharides of Vibrio cholerae O:1, serotypes Ogawa and Inaba. J Biol Chem. 1998;273(5):2777-83. PubMed PMID. doi: 10.1074/jbc.273.5.2777

31. Lullau E, Heyse S, Vogel H, Marison I, von Stockar U, Kraehenbuhl JP, Corthesy B. Antigen binding properties of purified immunoglobulin A and reconstituted secretory immunoglobulin A antibodies. J Biol Chem. 1996;271(27):16300-9. PubMed PMID. doi: 10.1074/ jbc.271.27.16300

32. Baranova DE, Levinson KJ, Mantis NJ. Vibrio cholerae O1 secretes an extracellular matrix in response to antibody-mediated agglutination. PloS One. 2018;13: e0190026. doi:10.1371/ journal.pone.0190026

33. Choi K-H, Schweizer HP. mini-Tn7 insertion in bacteria with single attTn7 sites: example Pseudomonas aeruginosa. Nat Protoc. 2006;1: 153-161. doi:10.1038/nprot.2006.24

34. Levinson KJ, Giffen SR, Pauly MH, Kim DH, Bohorov O, Bohorova N, Whaley KJ, Zeitlin L, Mantis NJ. Plant-based production of two chimeric monoclonal IgG antibodies directed against immunodominant epitopes of Vibrio cholerae lipopolysaccharide. J Immunol Methods. 2015;422:111-7. PubMed PMID: PMC4458452. doi: 10.1016/j.jim.2015.04.001

35. Schmitt-Ney M, Doppler W, Ball RK, Groner B. Beta-casein gene promoter activity is regulated by the hormone-mediated relief of transcriptional repression and a mammary-gland-specific nuclear factor. Mol Cell Biol. 1991;11: 3745-3755.

36. Rijnkels M, Kabotyanski E, Shore A, Rosen JM. The chromatin landscape of the casein gene locus. Horm Mol Biol Clin Investig. 2012;10: 201-205. doi:10.1515/hmbci-2012-0004

37. Yu X, Pollock D, Duval M, Lewis C, Joseph K, Meade H, Cavacini L. Neutralization of HIV by milk expressed antibody. J Acquir Immune Defic Syndr. 2013;62(1):10-6. PubMed PMID: PMC3531710. doi: 10.1097/QAI.0b013e318271c450

38. Klose KE. The suckling mouse model of cholera. Trends Microbiol. 2000;8: 189-191. doi:http://dx.doi.org/10.1016/S0966-842X(00)01721-2

39. Le Doare K, Holder B, Bassett A, Pannaraj PS. Mother's Milk: A Purposeful Contribution to the Development of the Infant Microbiota and Immunity. Front Immunol. 2018;9: 361. doi:10.3389/fimmu.2018.00361

40. Holmgren J, Hanson LA, Carlson B, Lindblad BS, Rahimtoola J. Neutralizing antibodies against Escherichia coli and Vibrio cholerae enterotoxins in human milk from a developing country. Scand J Immunol. 1976;5: 867-871. doi:10.1111/j.1365-3083.1976.tb03036.x

41. Holmgren J, Svennerholm AM, Ahrén C. Nonimmunoglobulin fraction of human milk inhibits bacterial adhesion (hemagglutination) and enterotoxin binding of Escherichia coli and Vibrio cholerae. Infect Immun. 1981;33: 136-141. 
42. Saha A, Rosewell A, Hayen A, MacIntyre CR, Qadri F. Improving immunization approaches to cholera. Expert Rev Vaccines. 2017;16: 235-248. doi:10.1080/14760584.2017.1249470

43. Severe K, Rouzier V, Anglade SB, Bertil C, Joseph P, Deroncelay A, Mabou MM, Wright PF, Guillaume FD, Pape JW. Effectiveness of Oral Cholera Vaccine in Haiti: 37-Month Follow-Up. Am J Trop Med Hyg. 2016;94(5):1136-42. PubMed PMID: PMC4856615. doi: 10.4269/ajtmh.15-0700

44. Sugimoto JD, Koepke AA, Kenah EE, Halloran ME, Chowdhury F, Khan AI, LaRocque RC, Yang Y, Ryan ET, Qadri F, Calderwood SB, Harris JB, Longini IM, Jr. Household Transmission of Vibrio cholerae in Bangladesh. PLoS Negl Trop Dis. 2014;8(11):e3314. PubMed PMID: PMC4238997. doi: 10.1371/journal.pntd.0003314

45. Watson AP, Armstrong AQ, White GH, Thran BH. Health-based ingestion exposure guidelines for Vibrio cholerae: Technical basis for water reuse applications. Sci Total Environ. 2018;613-614: 379-387. doi:10.1016/j.scitotenv.2017.08.297

46. Brandtzaeg P. Role of secretory antibodies in the defence against infections. Int J Med Microbiol. 2003;293: 3-15. doi:http://dx.doi.org/10.1078/1438-4221-00241

47. Murthy AK, Chaganty BK, Troutman T, Guentzel MN, Yu JJ, Ali SK, Lauriano CM, Chambers JP, Klose KE, Arulanandam BP. Mannose-containing oligosaccharides of non-specific human secretory immunoglobulin A mediate inhibition of Vibrio cholerae biofilm formation. PLoS One. 2011;6(2):e16847. PubMed PMID: PMC3036728. doi: 10.1371/journal. pone. 0016847 


\section{SUPPLEMENTARY MATERIALS}

\section{Supplementary Figures}

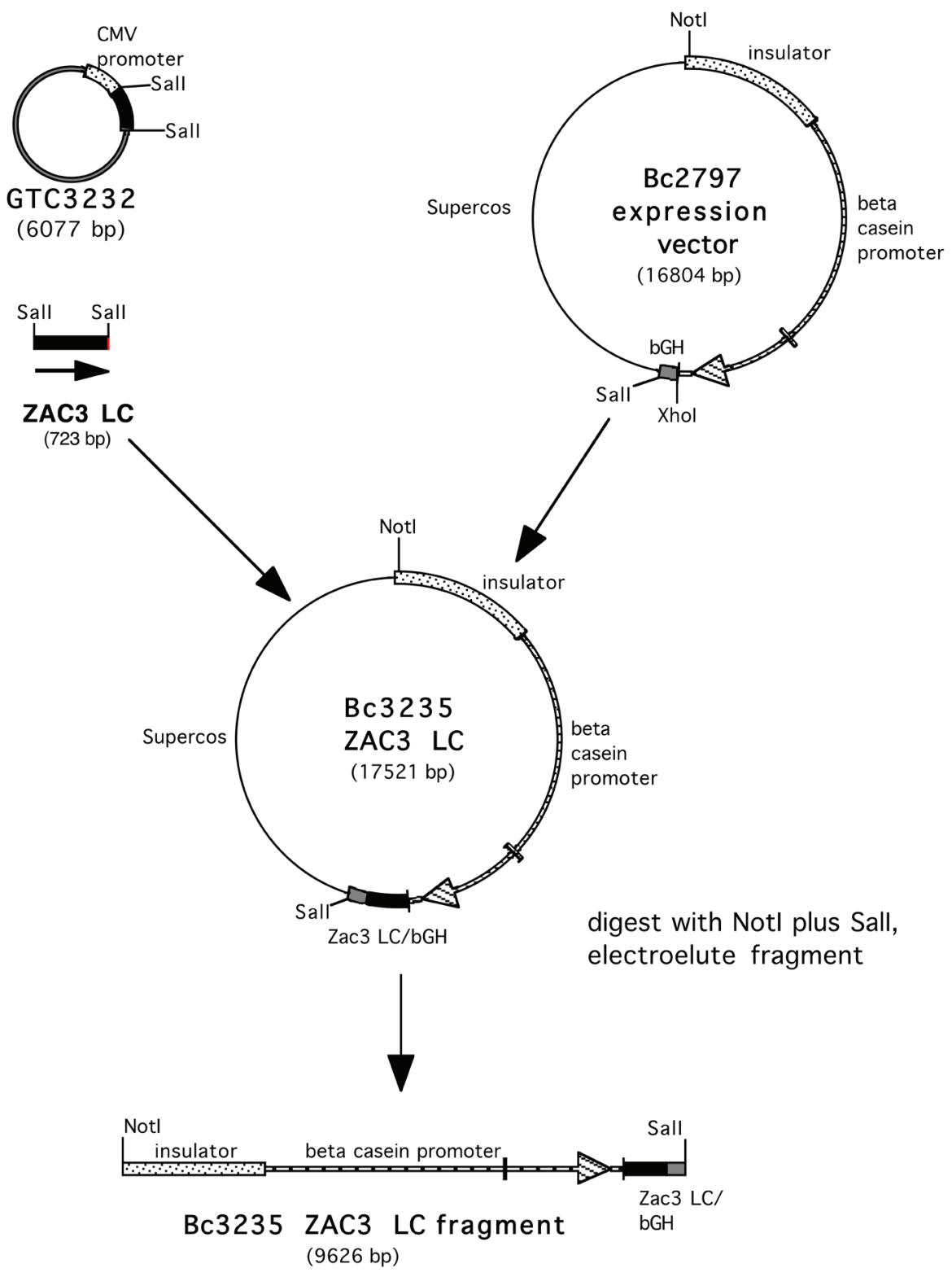

Supplementary Figure 1. Construction of BC3242 IgA1 HC. The sequence of the ZAC3 Vibrio cholerae antibody heavy chain was obtained from published sequences. The sequence was reverse translated, the DNA optimized, and synthesized by GeneArt, (Thermo Fisher). The HC sequence was obtained as a SalI $1.5 \mathrm{~kb}$ fragment in the mammalian expression vector pcDNA3-3238 HC. This SalI fragment was isolated and ligated into the XhoI site of the beta casein expression vector BC451 to yield BC3242 IgA1 HC. 


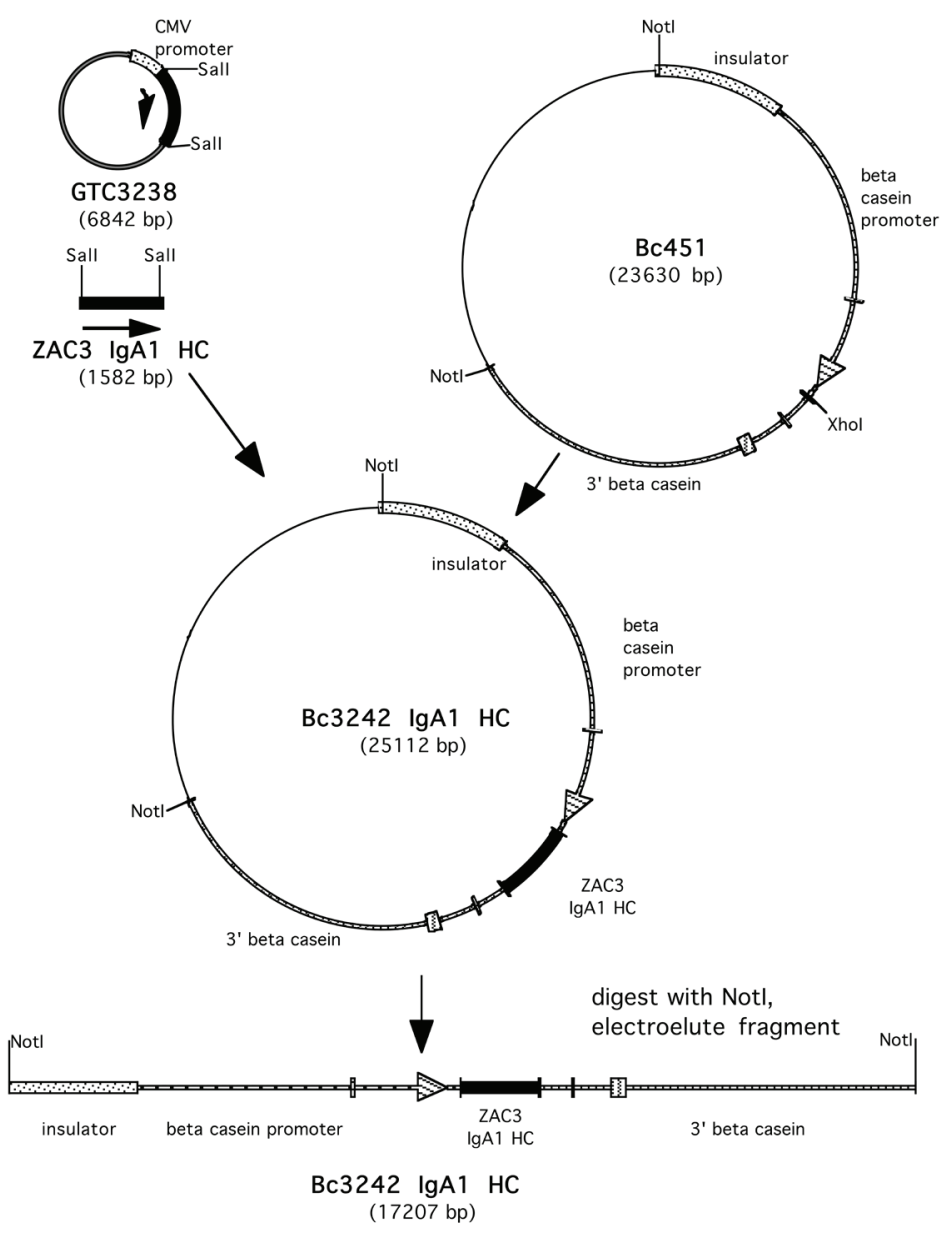

Supplementary Figure 2. Construction of BC3235 IgA1 LC. The VL sequence was also obtained from the published sequence, (with a 2 amino acid deletion at aa87). The protein was reverse translated, optimized for mammalian expression, and synthesized by GeneArt, (ThermoFisher), linked to the K constant region. The gene was present as a $500 \mathrm{bp}$ SalI fragment in the pcDNA3 expression vector BC3232. The 500 bp fragment was released by digesting with SalI and ligated into the XhoI site of BC2797 to yield BC3235 IgA1 LC. 


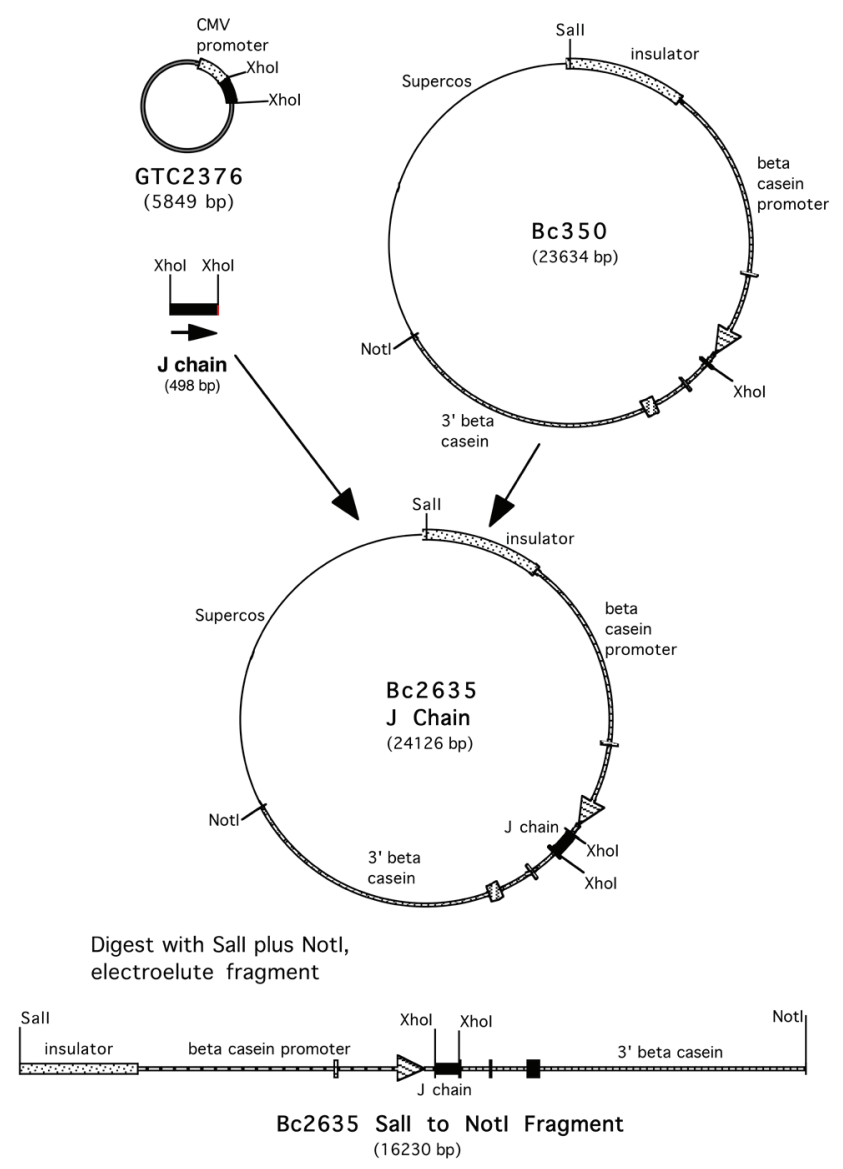

Supplementary Figure 3. Construction of BC2635 J chain. The J chain in the pcDNA3 vector, 2365 was obtained from L. Cavacini. It has the native sequence found in UniProtKB: P01591.4. The XhoI fragment containing the gene was cloned into the XhoI site of BC350 to yield BC2635 J Chain. 


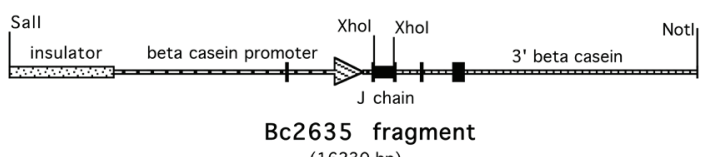

(16230 bp) ligate Bc2635 and Bc3235

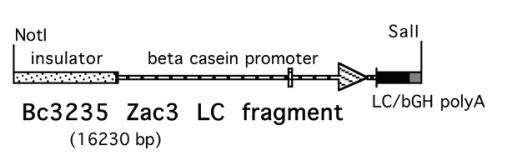

into SuperCos/Notl

using In vitro packaging (16230 bp)
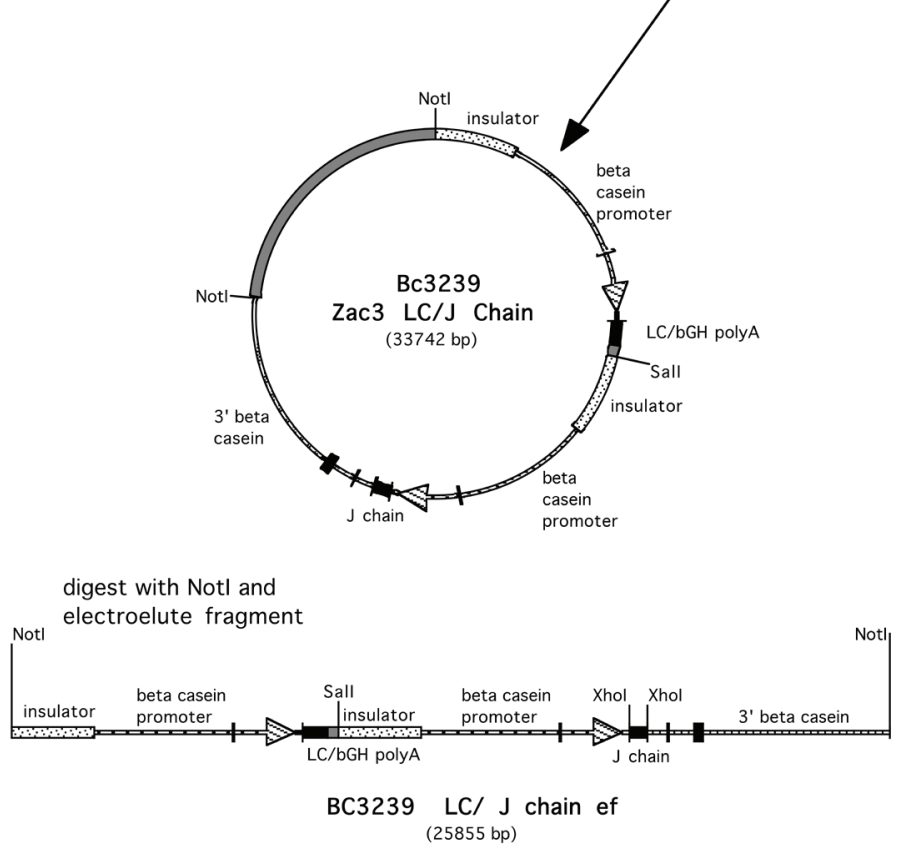

Supplementary Figure 4. Construction of BC3239 IgA LC/J Chain. To aid in micro-injection, the constructs carrying the LC and J chain were linked. The NotI-SalI fragment of BC3235 was isolated from the SuperCos bacterial vector. The SalI-NotI fragment containing the J chain was also isolated from BC2635. These 2 fragments were ligated together cloned into SuperCos using the lambda IVP system. The resulting plasmid, BC3239 carries both constructs as a $26 \mathrm{~kb}$ NotI insert. 

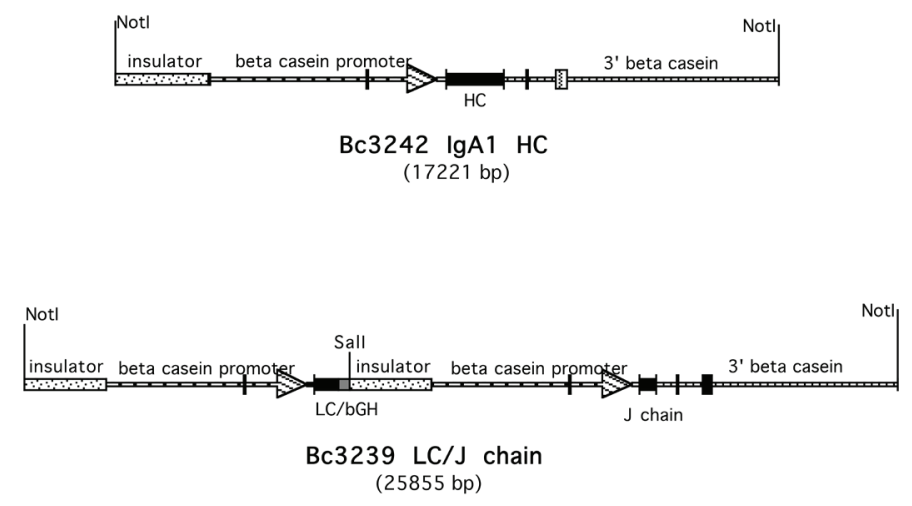

Supplementary Figure 5. Micro-injection Fragments BC3239 LC/J, BC3242 HC. The fragments for microinjection were isolated from the prokaryotic SuperCos vector by digesting the plasmids with restriction enzymes that flank the inserts. NruI, which cuts the SuperCos plasmid was also used. The Bc3239 LC/J chain and BC3242 HC were both digested with NotI, NruI, and the expression constructs were isolated by gel electrophoresis from the prokaryotic SuperCos fragments.

A.

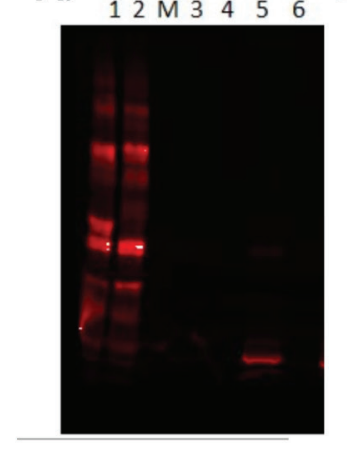

Anti- Kappa
B.

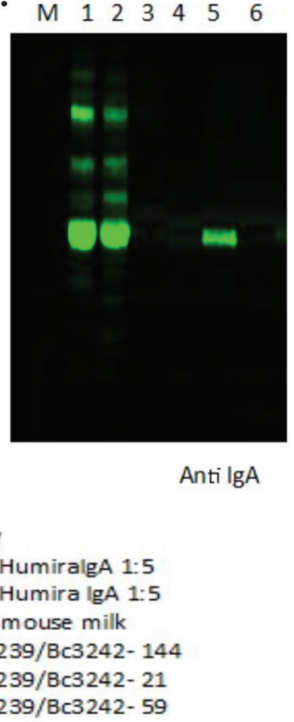

M. MW

390 HumiralgA 1:5

600 Humira IgA $1: 5$

Neg mouse milk

Вс3239/Вс3242- 144

5. ВС $239 /$ Bс3242- 21

6. BC3239/BC3242- 59

Anti IgA
C.

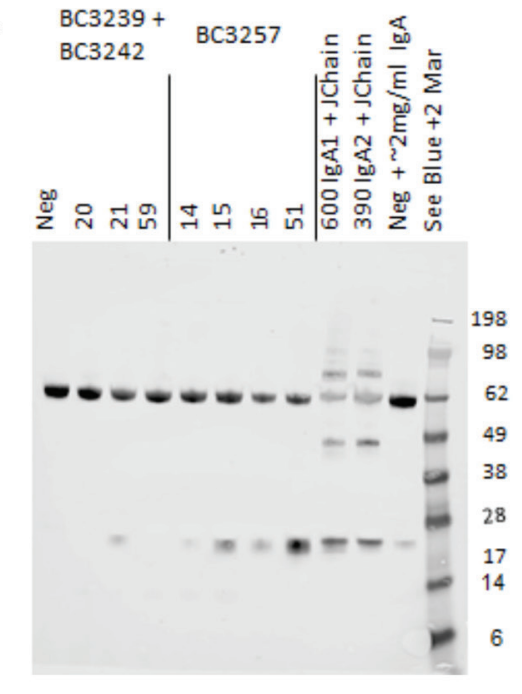

Supplementary Figure 6. Western blots confirm the presence of HC, LC, and J chain. Milk samples from control mice secreting HUMIRA IgA, previously unpublished, or ZAC-3 hIgA1 founder females, numbers 144, 21, 59 containing the BC3239 and BC3242 constructs were analyzed via western blot for (A) Kappa light chain and (B) human IgA. Lanes 1, 2, containing positive control Human IgA and lane 5, containing milk from founder female 21, exhibit highly positive signal for both Kappa LC and IgA. (C) Western blot showing that milk from mouse 21 is also positive for J-chain in comparison to positive controls. Data in (C) also includes preliminary findings from ZAC-3 IgA2 constructs, mouse numbers 14, 15, 16,51 , which were not pursued. ZAC-3 hIgA founder female 21 was subsequently bred. 
A.

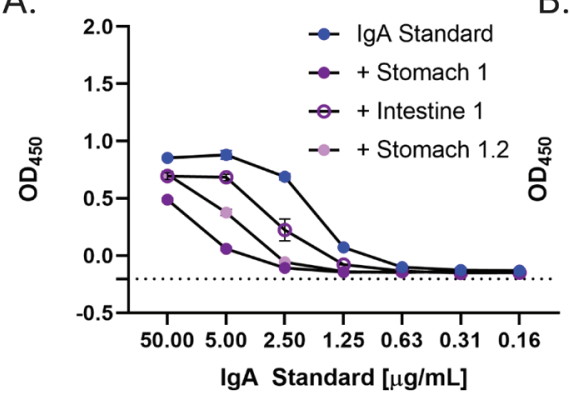

B.

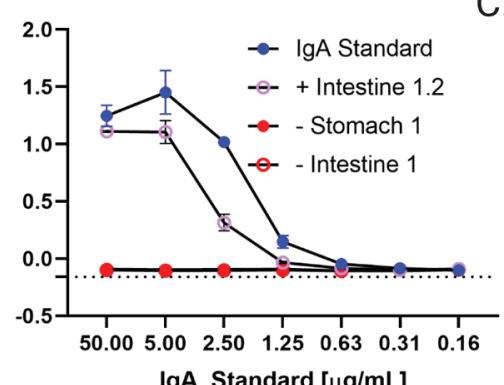

IgA Standard $[\mu \mathrm{g} / \mathrm{mL}]$
C.

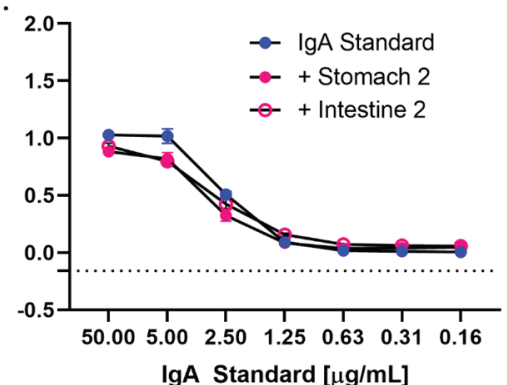

Supplementary Figure 7. Stomach and Intestinal Homogenates from Neonatal Mice Contain human IgA. Stomach and intestinal homogenates from pups suckled on ZAC-3 hIgA+ and hIgA- dams were analyzed by human IgA sandwich ELISA. Samples from a total of four pups were analyzed. A mouse suckled on an hIgA- dams (panel A, red symbols), two littermates (mice 1.1, 1.2) from a single hIgA+ dam (panels A, B) and a single mouse from a second litter (2.1; panel C). Human colostral IgA was used as a standard. mice Data are comprised of 1 biological replicate and 2 technical replicates.

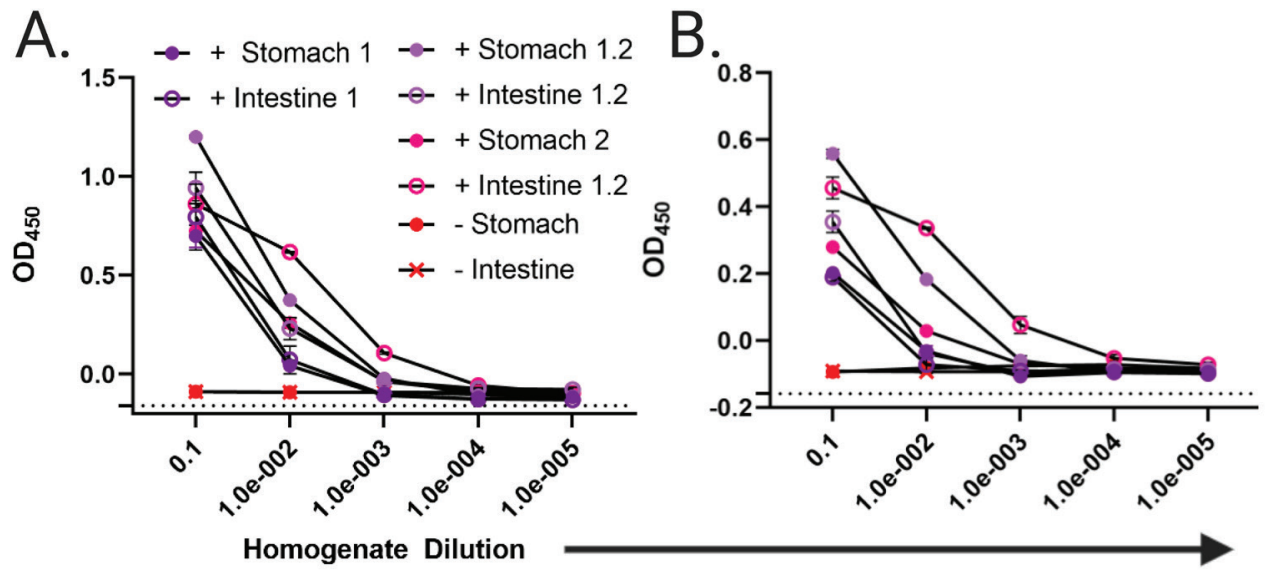

\section{Supplementary Figure 8. IgA in Stomach and Intestinal Homogenates Binds V. cholerae $\mathrm{O} 395$ and}

C6706. Whole cell ELISA with plates coated with either V. cholerae (A) classical Ogawa strain O395 or (B) El Tor Inaba strain C6706. Stomach and intestinal homogenates were diluted 1:10, and then 1:10 across the plate. Only homogenates from pups fed by ZAC-3 hIgA positive dams exhibit above background binding to whole V. cholerae cells. Labels are the same as in Figure S7. Again, homogenates from 1 pup fed from a WT dam were included as a reference. Each individual ELISA was conducted once with 2 technical replicates. 


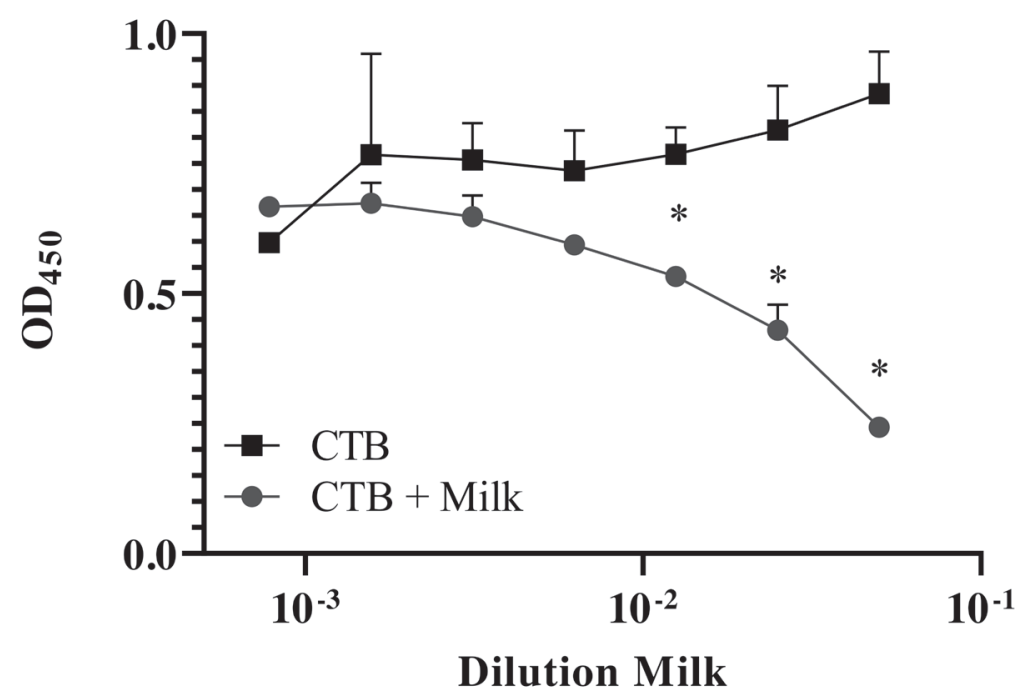

Supplementary Figure 9. Mouse Milk Competes with CT-B subunit for Binding GM-1. Cholera toxin B subunit (Millipore Sigma, Cat. No. C9972), at $10 \mu \mathrm{g} / \mathrm{mL}$, was mixed with WT milk (1:50 serial dilution, diluted 1:2 down the plate) for 1 hour, and then incubated with GM-1 coated ELISA plates for 1 hour (Sigma-Aldrich, Cat. No. 37758-47-7). There was a significant inhibition of CT-B binding to GM-1 in milk. Plates were developed as described in the materials and methods section. The experiment was performed in duplicate twice. $\left({ }^{\star} P<0.05\right)$. Significance was determined by 2 -way ANOVA followed by Sidak's multiple comparison test.

\section{Supplementary Videos Are Provided Via the Following Links} https://www.youtube.com/watch?v=W6TtMeEf5xA

\section{Supplementary Video 1. V. cholerae O1 strain O395 Remains Motile When Treated with} Control Milk. Mid-log phase V. cholerae O395 in LB were treated with a 1:100 dilution of control milk, from mouse 137 . Video is a compilation of 10 s videos, 100 total frames per video, played at 10 frames per second, captured at time 0 , and 5 minutes post treatment. Bacteria treated with control milk remain motile throughout the 5-minute time period, as evidenced by swirling bacteria coming into and out of focus. Video is representative of 3 technical replicates.

\section{https://www.youtube.com/watch?v=OddO2TXc8SA}

\section{Supplementary Video 2. V. cholerae O1 strain $\mathrm{O} 395$ Is Rendered Immobile When Treated} with Milk Containing ZAC-3 hIgA1. Mid-log phase V. cholerae O395 in LB were treated with a 1:100 dilution of milk containing ZAC-3 hIgA, from mouse 172. Video is a compilation of 10 s videos, 100 total frames per video, played at 10 frames per second, captured at time 0 , and 5 minutes post treatment. Bacteria treated with ZAC-3 hIgA-containing milk do not exhibit flagellar-based motility throughout the 5-minute time period, as evidenced by lack of swirling bacteria coming into and out of focus. Video is representative of 3 technical replicates. 
https://www.youtube.com/watch?v=V-k0fKW735U

\section{Supplementary Video 3. V. cholerae O1 strain O395 Remains Motile When Treated with} Stomach Homogenates from Control Pups. Mid-log phase V. cholerae O395 expressing a plasmid with mCherry in LB were treated with a 1:100 dilution of stomach homogenates from a 4- to 5 -day- old pup nursed from a control, WT CD-1 dam. Video is a compilation of 10s videos, 10 total frames per video, played at 10 frames per second, captured at time 0 , and 5 minutes post treatment. Bacteria treated with control homogenates remain motile throughout the 5-minute time period, as evidenced by swirling bacteria coming into and out of focus. Video is representative of 11 individual experiments with stomach homogenates from pups fed from 6 WT CD-1 dams.

\section{https://www.youtube.com/watch?v=s1PLyMxtOUM}

\section{Supplementary Video 4. V. cholerae O1 strain $\mathrm{O} 395$ Is Rendered Immobile When Treated} with Stomach Homogenates Containing ZAC-3 hIgA1. Mid-log phase V. cholerae O395 expressing a plasmid with mCherry in LB were treated with a 1:100 dilution of stomach homogenates from a 4- to 5-day-old pup nursed from a transgenic CD-1 dam that secretes ZAC-3 hIgA in milk. Video is a compilation of 10 s videos, 10 total frames per video, played at 10 frames per second, captured at time 0 , and 5 minutes post treatment. Bacteria treated with ZAC-3 hIgA-containing homogenates exhibit less flagellar-based motility throughout the 5-minute experiment, as shown by quantitation of the number of moving bacteria in Figure 5B. Video is representative of at least 3 individual experiments with stomach homogenates from pups fed from at least 2 ZAC-3 hIgA1 CD-1 dams.

\section{SUPPLEMENTARY TABLES}

\begin{tabular}{ccc}
\hline Supplementary Table 1 . List of bacterial strains utilized in this work. \\
\hline Strain/Name & Characteristics/Sequence & Source/Reference \\
\hline V. cholerae O395 & Wild-type Classical Ogawa & John Mekalanos (Harvard Medical School) \\
V. cholerae C6706 & Wild-type El Tor Inaba & Christopher Waters (Michigan State Univ.) \\
DB364 & V. cholerae O395 pGW104 & This work \\
\hline
\end{tabular}

\begin{tabular}{ccc}
\hline \multicolumn{2}{c}{ Supplementary Table } & 2. List of primers utilized in this work. \\
\hline Name & Sequence & Source/Reference \\
\hline BC3242 FWD & CCGTGACTTGGAGCGAATCT & This work \\
BC3242 REV & GCGTCCTGAGAAGGTGGG & This work \\
BC3239 FWD & GACCTGGCCGAGTACTTCTG & This work \\
BC3239 REV & GTCCTCTTGATTTCCAGCTTGGT & This work \\
PA1/04/03_F_Kpn1 & ATA GGT ACC ATT TAT CAG GGT TAT TGT CTC & This work \\
& ATG A & This work \\
PA1/04/03_ORF_SOE_ & CCT TGC TCA CCA TGC TTA ATT TCT CCT CTT & \\
R1 & TAA TTC TAG ATG TG & This work \\
xFP_ORF_SOE_F2 & ATT AAA GAG GAG AAA TTA AGC ATG GTG AGC &
\end{tabular}


mcherry_R_hindIII

TTA AAG CTT GCA TGC CTG CAG ACT AGT CTA

This work

CT

\begin{tabular}{|c|c|c|}
\hline \multicolumn{3}{|c|}{ Supplementary Table 3. List of plasmids utilized in this work. } \\
\hline Plasmid Name & Characteristics/Sequence & Source/Reference \\
\hline $\mathrm{pBC1}$ & Contains goat $\beta$-casein expression cassette & Invitrogen \\
\hline BC451 & $\begin{array}{c}\text { Beta Casein expression vector encoding a } 7.2 \mathrm{~kb} \\
\text { downstream sequence including the last } 3 \text { exons of } \\
\text { beta casein }\end{array}$ & (X. Yu et al. 2013) \\
\hline BC350 & $\begin{array}{c}\text { Beta Casein expression vector encoding a } 7.2 \mathrm{~kb} \\
\text { downstream sequence including the last } 3 \text { exons of } \\
\text { beta casein }\end{array}$ & (X. Yu et al. 2013) \\
\hline BC2797 & $\begin{array}{l}\text { Beta Casein expression vector encoding a 300bp bgH } \\
\text { polyA sequence }\end{array}$ & (X. Yu et al. 2013) \\
\hline BC3242 & BC451 encoding ZAC-3 IgA1 HC (Figure S1) & This work \\
\hline BC3235 & BC2797 encoding ZAC-3 IgA1 LC (Figure S2) & This work \\
\hline BC2635 & BC350 encoding J chain (Figure S3) & This work \\
\hline BC3239 & $\begin{array}{l}\text { NotI-SalI fragment of BC3235 and SalI-NotI fragment } \\
\text { of BC2635 ligated into Supercos (Figure S4) }\end{array}$ & This work \\
\hline pMQCherry80 & $m$ Cherry open reading frame & $\begin{array}{l}\text { Matthew Wargo, unpub- } \\
\text { lished }\end{array}$ \\
\hline pUC18-mTn7T-eyfp-Gm & constitutive derivative of the $P_{l a c}$ promoter $\left(P_{A / 01 / 04 / 03}\right)$ & [33] \\
\hline pGW104 & $\begin{array}{l}\text { pUC18T-mTn7T-eyfp with constitutive } \mathrm{P}_{\text {lac }} \text { promotor } \\
\text { and mCherry ORF }\end{array}$ & This work \\
\hline
\end{tabular}

\section{FOOTNOTES}

Submitted March 27, 2020 | Accepted April 14, 2020 | Published May 8, 2020

\section{COPYRIGHT}

Copyright () 2020 Pathogens and Immunity.

This is an open-access article distributed under the terms of the Creative Commons Attribution 4.0 International License. 